\title{
A Semiparametric Model for Bond Pricing with Life Cycle Fundamental ${ }^{* \dagger}$
}

\author{
Zongwu Cai $^{a}$ Jiazi Chen $^{b}$ Linlin $\mathrm{Niu}^{b, c}$ \\ ${ }^{a}$ Department of Economics, University of Kansas, Lawrence, KS 66045, USA

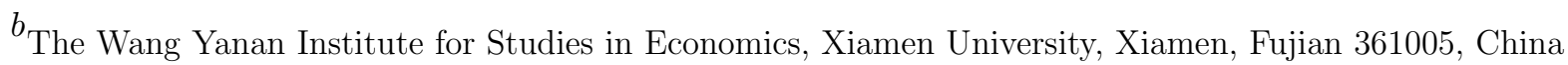 \\ ${ }^{c}$ MOE Key Lab of Econometrics and School of Economics, Xiamen University, Xiamen, Fujian 361005, China
}

January 7, 2021

\begin{abstract}
It is well documented in the literature that individual saving decisions vary with the life cycle and consequently, a changing demographic age structure affects aggregated savings, which then drives a slow movement of interest rates. In this paper, we propose a semiparametric affine arbitrage-free yield curve model with a lowfrequency trend structure driven by the entire age distribution through a life cycle impact function. The unified framework not only fully explores the demographic age structure to robustly explain yield trend, but also utilizes efficiently the yield curve to infer the S-shaped age impact function of the whole life cycle. We estimate the model with quarterly U.S. data from 1950s to present. The results show remarkable in-sample fit and out-of-sample outperformance with respect to popular alternative models. After removing the demography-driven trend especially pertaining to the baby boomers life cycle, the remaining term structure component is stationary with counter-cyclical risk premia.
\end{abstract}

Keywords: Demographic distribution, Life cycle, Term structure models, Semiparametric model, Functional data analysis.

JEL Classification: E43, G12, J11

*The authors thank Thomas Sargent and Harald Uhlig for their valuable comments, and thank seminar and workshop participants at Xiamen University, Zhejiang University, National Chung Hsing University, Monash University, Hunan University, Southwestern University of Finance and Economics, Shanghai Academy of Social Science, and Northeastern University of Finance and Economics. This research is partly supported by the National Natural Science Foundation of China with grant numbers 71631004, 71871193 and 72033008 .

${ }^{\dagger}$ Corresponding author: Linlin Niu, Rm A306, Economics Building, Xiamen University, Xiamen, 361005, Fujian, China. Email: llniu@xmu.edu.cn. Phone: +86-592-2182839. Fax: +86-592-2187708. 


\section{Introduction}

High persistence (trending) is a well-known stylized fact on the dynamics of interest rates. Technically, modeling this trending feature is critical for predicting yields and understanding risk premia in practice. Theoretically, understanding the fundamental mechanism behind the trend is of great importance for macroeconomic research, which also has crucial implications for investment strategies and economic policy making. On the technical side, various methods have been proposed to address this persistent feature, many of which effectively lead to better prediction and produce more meaningful measures of risk premia. On the fundamental side, however, the economic mechanism is still open to deeper investigation. This paper links the persistence with economic fundamentals of demographic age distribution through life cycle impact on yields within a unified modeling framework. The model demonstrates empirical power on forecasting accuracy and provides remarkable insights to various macro-finance issues.

Figure 1 displays a three-dimensional plot of the U.S. Treasury yield curve from 1952:Q2 to 2018:Q1. Viewed from this life-long span of nearly seven decades, the business cycle fluctuations, which usually lasts for a few years up to a decade or so, seem to be doomed by the most evident large swing starting from a few percents in the 1950s, climbing up all the way to double digits high in the 1970s and 1980s, and then sliding down for more than three decades until the recent era of "zero lower bound (ZLB)". Also, in view of this long span, the persistence is hard to be justified by a mean-reverting process with a constant long-run mean, at least within the interval of a few decades. From the relationship between yields and forward spreads in a long span starting from 1952, Fama (2006) also points out a strong evidence to suggest a nonstationary long-term mean and local mean-reverting process at the business cycle length.

In the vast literature on interest rate and yield curve modeling, however, the majority rely on the assumption of constant long-run mean, ever since the pioneering work on noarbitrage dynamic term structure models initiated by Vasicek (1977) and Cox et al. (1985), in particular, the reduced-form popularized by Diebold and Li (2006) or with a macrofinance perspective starting from Ang and Piazzesi (2003). A possible misspecification 


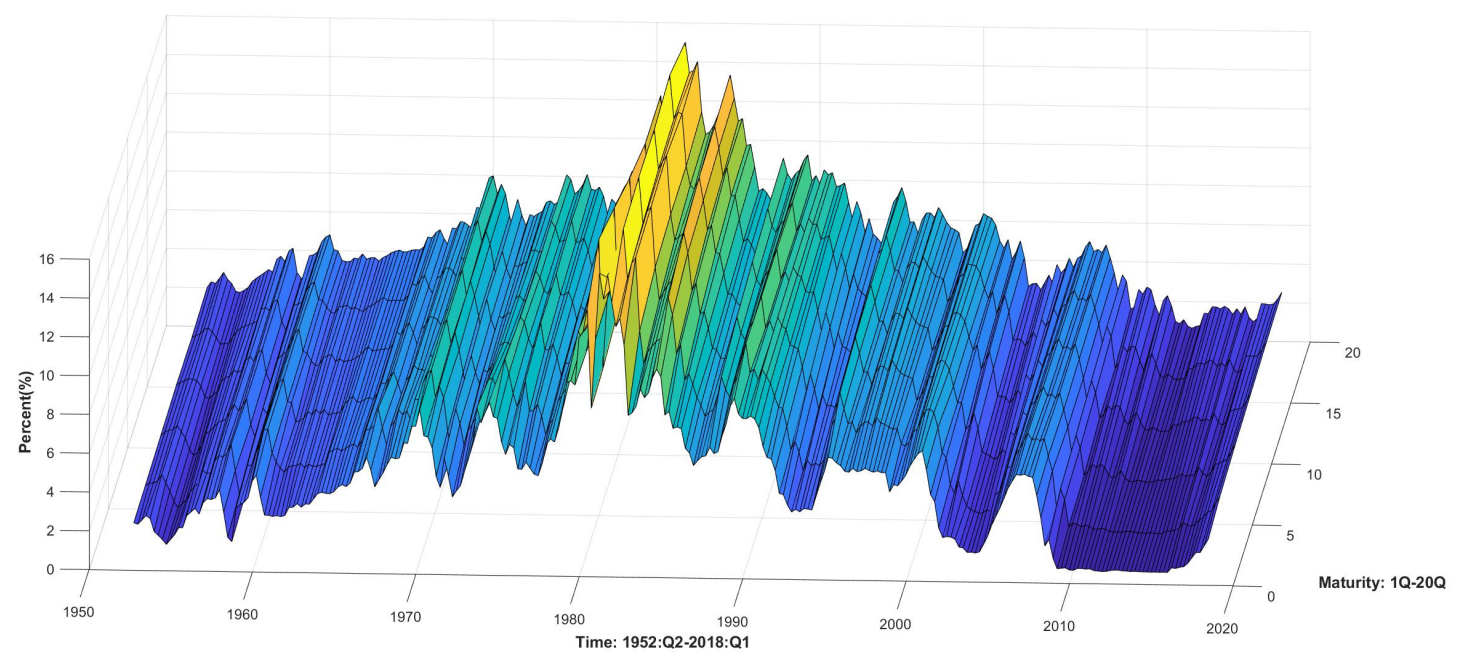

Figure 1. U.S. Treasury yield curve from 1952:Q2 to 2018:Q1

on the trending behavior may lead to various undesirable consequences. In yield curve forecasting, out-of-sample prediction may be systematically biased. Estimating a dynamic yield model with autoregressive process of constant mean tends to under predict if yields in the chosen sample are trending up, or over predict along a downward trend. Since long term yields are risk adjusted average of future short-term rate, under prediction (over prediction) on future short-term rate results in overpricing (underpricing) of risk premia. In macro-finance study, the misspecification of long run mean of short rates is likely to cause estimation bias on the economic mechanism behind interest rate determination. For example, in a Taylor rule setting, monetary policy reacts to output gap and inflation. When these variables have stochastic trends, an empirical Taylor rule assuming constant mean tends to introduce bias into the estimated policy coefficients.

Therefore, the drawbacks of trend misspecification in yields have called for research attention to introduce time-varying means into the state dynamics. One approach is to employ long memory processes to describe the state dynamics, such as cointegrated vector autoregression in reduced-form as in Bowsher and Meeks (2008) or with no-arbitrage restrictions as argued in Goliński and Zaffaroni (2016) and Bauer and Rudebusch (2020), which are shown to outperform random walk in short horizons or alternative short-memory models. An alternative approach is to introduce regime switching as in Ang and Bekaert (2002), though it is not flexible enough to accommodate a sequence of permanent shocks 
addressed in Fama (2006). Adaptive autoregressive model is another way to reflect the global time-varying mean using local stationary process with flexibility, parsimony, and desirable forecast property studied by Chen and Niu (2014). Introducing external persistent measures beyond yield information also provides a way to proxy the trending behavior of yields as in Cieslak and Povala (2015).

In the strand of macro-finance term structure literature, efforts have been made on investigating the fundamental sources of stochastic trend in yields. According to the Fisherian equation, trends in inflation and real interest rate become natural candidates of the stochastic trend of nominal interest rate. For example, Cieslak and Povala (2015) take the discounted moving average of past inflation to proxy the adaptive expectation of trend inflation as the major source of trend in yields and find that the resulting cyclical component in yields can well predict short-rate changes as well as excess bond returns, whereas Bauer and Rudebusch (2020) find that both "falling stars", the falling trend in inflation during 1980s and 1990s and the falling trend in real interest rate during the last two decades, are relevant to explaining the persistent decline of nominal yields over the last forty years. Yet, the drivers behind the falling stars are still open to questions.

This paper attempts to build a joint model of nominal yields with stochastic trends and local mean-reverting process based on a deeper economic foundation in a unified framework, where the trend driver is the demographic age distribution through the life cycle impact functions on the real rate and inflation, respectively, which then determine the impact function on nominal yields.

Relationships between population age structure and real rate and inflation have been studied in the literature both on theory and empirical evidence. Indeed, Modigliani (1966) gives an analytical foundation on the life cycle impact of demographic age structure on real rate through aggregate savings. The life-cycle theory on savings predicts that when population growth exceeds the stationary level for a successive year, the bigger proportion of these cohorts of baby boomers in the population will lead to higher ratio of working-aged households when they are in their wealth accumulation phase, thus a positive net aggregate saving; later in their de-saving phase, the same cohort leads to a higher ratio of older households, thus a negative net aggregate saving. Excess savings tend 
to decrease interest rate and asset returns, and shortage of savings tend to increase returns. Moreover, population aging and longevity risk tend to increase saving and prolong the saving phase before retirement (Bloom et al., 2003; Zhang et al., 2003; Cocco and Gomes, 2012). Both population growth and increasing life expectancy of the baby boomers can be transferred to lower real rate during their working ages. In the U.S.A., the baby boom after the World War II up to the 1960s and increasing life expectancy have a significant impact on the macro economy and asset returns (Bakshi and Chen, 1994; Abel, 2003; Goyal, 2004; Krueger and Ludwig, 2007; DellaVigna and Pollet, 2007; Favero et al., 2011). Also, international studies confirm a similar pattern (Ang and Maddaloni, 2005; Aksoy et al., 2019). On the other hand, although less extensively studied than the demographic link to real rate via savings, there are empirical evidences on the correlation between changing demographic structure and inflation in the U.S.A. (McMillan and Baesel, 1990) and OECD countries (Lindh and Malmberg, 2000; Juselius and Takáts, 2015), and possible mechanisms of fiscal redistribution across generations have been proposed to explain the phenomena (Bullard et al., 2012; Katagiri et al., 2020).

To visualize the bulky weight and possible impact of the baby boomers in the population and their slow movement along time, the annual data of U.S. demographic age distribution is plotted in Figure 2, which can be downloaded from the U.S. Census Bureau, covering the number of each age bin from below one year to age 85 and above from 1940 to 2018. At each point in time, the weight of each cohort is computed with respect to the total population. As shown in Figure 2, the higher weight of the baby boomer is the most evident feature along both dimensions: along time, the baby boomers stand out as a surge of infant weight at the lower edge of the age distribution after the World War II for about twenty years; then as they live through different life stages, these cohorts form a persistent wave-like pattern across the age distribution. A resulting implication is that their economic behavior counts more in the aggregate level; in turn, their changing behavior along the life cycle may slowly move macroeconomic variables, including asset returns.

Based on the discussions in the literature on the life-cycle mechanisms affecting aggregate variables, it follows evidently that the demographic age impact functions on real rate 


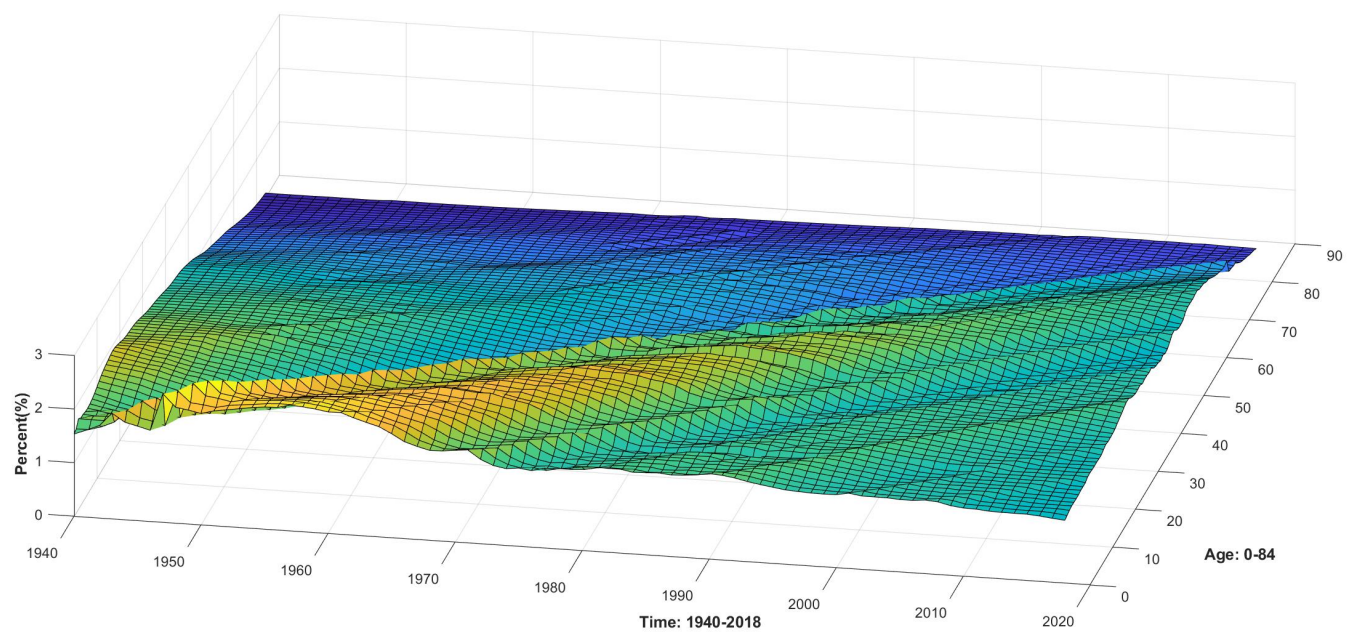

Figure 2. Dynamics of the U.S. demographic age distribution

and inflation add up to the impact function on nominal yields. This paper shows that the age impact function on yields can be strongly identified from the term structure of interest rates under the expectation hypothesis (EH). Since the demographic structure is slow moving with very low uncertainty in predicting the demographic survival rates in normal time, age distributions in future horizons up to a few years can be precisely projected, such that the age distribution impact on long-term yields is the average of its impacts on future short-term yields, thus the EH is justified. Augmenting a typical stationary and mean reverting yield component to the low-frequency and demography-driven trend under functional coefficients, a novel no-arbitrage term structure model is derived in this paper.

Our work contributes to the existing macro-finance studies in the following four aspects. First, our model provides a unified framework to explain the time-varying mean of yield curve through a life-cycle fundamental driving both the trends of real rate and inflation. The joint process of slow-moving trend and mean-reverting component not only produces good in-sample fit, but also forecasts out-of-sample remarkably well, especially for median- to long-term yields and at the median- to long-term forecast horizons. The rich information from the whole yield curve helps robust inference on the natural rate and provides complementary policy recommendation derived from dynamic stochastic general equilibrium models relying on limited macroeconomic sample information (Eggertsson 
et al., 2019). Compared to the moving average of inflation trend proxy used in Cieslak and Povala (2015), our extracted trend for long-term yields is forward-looking and incorporates the trends in both inflation and real rate. Based on a consistent Fisherian relationship to link the trend in short-term nominal rate as the sum of trends in short-term real rate and inflation, our model provides a more intuitive spanning mechanism driving the "falling stars" from the short- to long-term horizons through expectation, as compared to the latent unspanned mechanism in Bauer and Rudebusch (2020), where agents are assumed to first learn the "shifting endpoint" in the long run and then determine backwards the common movement in yields (Kozicki and Tinsley, 2001).

Second, our approach of introducing the whole age distribution in a functional form to explain yield trend is more efficient and robust with consistent interpretation than the single index approach of summarizing demographic structure with a simple indicator. While our framework is somewhat close to Favero et al. (2016), in the sense that they introduce an index of age composition, termed as an MY ratio between middle-aged (40$49)$ to young (20-29) population as a proxy for the demographic structure (Geanakoplos et al., 2004), and derive a no-arbitrage term structure model based on the argument that the short rate follows a Taylor rule targeting on a time-varying demographic factor. However, the single index approach might suffer from some drawbacks. It is not robust to different compositions of age cohort and the resulting empirical evidence is mixed in someway (Poterba, 2001) and fails to explain the persistently low rate in the recent decade (Del Negro et al., 2019). It is also inefficient without utilizing the whole age distribution. Like many life-cycle analyses, the information on younger age cohort before entering the labor force is missing from the MY ratio. To the extent of consuming accumulated saving of the depending family, younger cohort may have an important impact on the household's economic and portfolio decisions (Browning and Ejrnæs, 2009; Love, 2010). Thus, an unusually larger or smaller proportion of younger cohort should affect aggregate savings and asset returns, which can not be captured by a MY ratio.

Third, methodologically, our framework of the term structure of interest rates helps to infer the life-cycle impact function of age distribution with strong identification. In existing research, the impact function of the demographic age distribution was estimated 
commonly for various macroeconomic variables in a single equation setting; see, for example, Fair and Dominguez (1991), Higgins (1998), Park et al. (2010), and Park (2010) for details. However, due to limited sample of typical macroeconomic time series, the parameter inference and specification choice are subject to uncertainty from small sample size. The quantitative effects of demographic structure on the macro economy is inconclusive with much uncertainty on the implied equilibrium level of macroeconomic variables and policy recommendations. Imposing the cross-equation restrictions from the term structure under the $\mathrm{EH}$ and no-arbitrage restrictions helps to resolve the uncertainty to a large extent, such that the estimation results on the life-cycle impact function are more reliable with higher statistical significance. Our empirical results not only provide a consistent evidence on the life-cycle driven large swing of the U.S. yields during the last seven decades, but also demonstrate remarkable accuracy in out-of-sample yield forecast on long horizon compared to results from a bunch of alternative popular models.

Finally, technically, the model is set up in a semiparametric econometric representation under no-arbitrage conditions. It shows that the functional impact of the demographic age distribution can be approximated nonparametrically by using the Fourier flexible expansion (FFE) proposed by Park et al. (2010) not only in the single equation of the short rate, but also can be extended to long-term yields with cross-equation restrictions under the no-arbitrage condition. The cross-equation restrictions help to identify the underlying functional form with higher significance than in a single equation setting. Compared to previous semiparametric dynamic term structure model without macroeconomic foundation or theoretical restrictions, such as the models in Ghysels and Ng (1998) and Härdle and Majer (2016), our model demonstrates the potential of introducing the nonparametric method to assist structural macro-finance analysis. Furthermore, to estimate the parameters in the cyclical component, a nonlinearity in the factor loadings of measurement equations makes the estimation challenging both on the searching for global optimum and on learning parameter uncertainties. To overcome this difficulty, we use the Bayesian approach of Markov chain Monte Carlo (MCMC) method to estimate the proposed model.

The rest of the paper is arranged as follows. Section 2 presents empirical evidence of the age distribution impact on real rate, inflation, and short term nominal yield. Section 
3 derives the no-arbitrage interest rate term structure model with a time-varying mean driven by the demographic age distribution. Section 4 describes the estimation strategy with Bayesian method. Section 5 discusses the empirical results of the model and shows its forecast performance in yields. Section 6 concludes the paper.

\section{Empirical Evidence of Age Distribution Impact on Yields}

This section presents the single equation evidence on the age impact function from separately regressing real rate $r_{t}$, inflation $\pi_{t}$, and nominal short-term yield $i_{t}$ on the demographic age distribution.

\subsection{Trend Decomposition with a Functional-Form Demographic Factor}

It is assumed that each of these economic variables, named as $x_{t}$ for general elaboration, is composed of a time-varying long-run trend $x_{t}^{*}$, and a cyclical component $\tilde{x}_{t}$, which reverts to the long-run mean $x_{t}^{*}$ as

$$
x_{t}=x_{t}^{*}+\tilde{x}_{t}
$$

and that the time-varying trend $x_{t}^{*}$ is driven by the demographic age distribution through its impact $g(s)^{x}$ as follows

$$
x_{t}^{*}=\int_{\underline{\mathrm{S}}}^{\bar{S}} f_{t}(s) g^{x}(s) d s,
$$

which is a functional data analysis model; see, for example, Rasay and Silverman (1997)

and Park et al. (2010) for details, where the age span is $s \in[\underline{S}, \bar{S}], f_{t}(s)$ denotes the density function of demographic distribution at time $t$, and $g^{x}(s)$ represents the age impact function related to the variable $x_{t}$. Then, by the Fisher equation relating the short term nominal rate as the sum of real rate and inflation,

$$
i_{t}=r_{t}+\pi_{t}
$$


it is reasonable to assume that the Fisherian relationship should hold for both their timevarying long-run means and cyclical terms,

$$
i_{t}^{*}=r_{t}^{*}+\pi_{t}^{*} \quad \text { and } \quad \tilde{\imath}_{t}=\tilde{r}_{t}+\tilde{\pi}_{t}
$$

Then, based on common age factor $f_{t}(s)$ and the functional form of (2.1), the implication on the impact functions is decomposed as

$$
g^{i}(s)=g^{r}(s)+g^{\pi}(s)
$$

Hence, the demographic age impact functions on real rate and inflation add up to the impact function on nominal yields.

Next, our turn is to estimate the impact function $g(s)$. Indeed, to this end, there are many methods available in the literature such as a series method; for example,

$$
g(s)=\sum_{i=1}^{\infty} \alpha_{i} \psi_{i}(s)
$$

where $\left\{\psi_{i}(s)\right\}$ is a sequence of (orthogonal) basis functions. For simplicity, motivated by the idea in Park et al. (2010), we propose using a semiparametric method to approximate $g(s)$ with a Fourier flexible series as

$$
g_{\kappa}(s)=\sum_{i=1}^{\kappa} \alpha_{i} \psi_{i}(s)=\alpha_{1}+\alpha_{2} s+\alpha_{3} s^{2}+\sum_{j=1}^{J}\left[\alpha_{4, j} \cos (j s)+\alpha_{5, j} \sin (j s)\right]
$$

where $\kappa=3+2 J \rightarrow \infty$, denoting the number of parameters in the approximating function $g_{\kappa}(s)$, by assuming that $\left\|g_{\kappa}(s)-g(s)\right\|_{2} \rightarrow 0$ as $\kappa \rightarrow \infty$.

Remark 1. Since the age impact function contains the trigonometric series, it is desirable to scale the age span into the interval $[0,1]$. That is, with a given common support $s \in[\underline{S}, \bar{S}]$ for $f_{t}(s), f_{t}(s)$ is transformed by $f_{t}^{*}\left(s^{*}\right)=f_{t}\left[\underline{S}+(\bar{S}-\underline{S}) s^{*}\right]$, such that $f_{t}^{*}(s)$ has the common support $s^{*} \in[0,1]$. The original impact function $g$ with respect to $f_{t}$ can be recovered from the impact function $g^{*}$ with respect to $f_{t}^{*}$ by the transformation $g(s)=g^{*}[(s-\underline{\mathrm{S}}) /(\bar{S}-\underline{\mathrm{S}})]$.

The reason for choosing the FFE method is that as illustrated in Park et al. (2010), 
this FFE approximation might be more parsimonious than other parametric methods which often suffer from the typical multicollinearity problem, and it has an intuitive interpretation on the selected Fourier series at different frequencies. It is also more efficient with an asymptotic distribution, as shown in Andrews (1991), compared to other methods such as the parametric polynomial approximation in Fair and Dominguez (1991). Thus, plugging (2.2) into (2.1) leads to

$$
x_{t}^{*}=\int_{0}^{\bar{S}} f_{t}(s) g(s) d s \approx \sum_{i=1}^{\kappa} \alpha_{i} \int_{0}^{\bar{S}} f_{t}(s) \psi_{i}(s) d s .
$$

Next, by dividing the age support $[0, \bar{S}]$ into $M$ age sub-intervals, $\left[0, s_{1}\right), \ldots,\left[s_{m-1}, s_{m}\right)$, $\ldots,\left[s_{M-1}, \bar{S}\right]$, each with a weight

$$
w_{t}\left(s_{m}\right)=\int_{s_{m-1}}^{s_{m}} f_{t}(s) d s
$$

then, the integral in (2.3) can be approximated as

$$
\int_{0}^{\bar{S}} f_{t}(s) \psi_{i}(s) d s \approx \sum_{m=1}^{M} w_{t}\left(s_{m}\right) \psi_{i}\left(\bar{s}_{m}\right)
$$

with $\bar{s}_{m}=\left(s_{m-1}+s_{m}\right) / 2$.

\subsection{Linear Estimator}

Given the above approximation $(2.4),(2.3)$ can be written as the following simple linear form

$$
x_{t}^{*}=\alpha^{\prime} z_{t}
$$

where $\alpha=\left(\alpha_{1}, \cdots, \alpha_{\kappa}\right)^{\prime}$ and $z_{t}=\left(z_{t 1}, \cdots, z_{t \kappa}\right)^{\prime}$ with $z_{t i}=\sum_{m=1}^{M} w_{t}\left(s_{m}\right) \psi_{i}\left(\bar{s}_{m}\right)$ for $1 \leq$ $i \leq \kappa$. Given $x_{t}$ and $z_{t}, \alpha$ can be estimated with an OLS estimator $\widehat{\alpha}$, from which the age impact function can be inferred as

$$
\widehat{g_{\kappa}}\left(\bar{s}_{m}\right)=\widehat{\alpha}^{\prime} \Psi\left(\bar{s}_{m}\right)
$$

where $\Psi\left(\bar{s}_{m}\right)=\left(\psi_{1}\left(\bar{s}_{m}\right), \cdots, \psi_{\kappa}\left(\bar{s}_{m}\right)\right)^{\prime}$ evaluated at $\bar{s}_{m}$ for $1 \leq m \leq M$. While the $\kappa \times \kappa$ variance-covariance matrix of $\widehat{\alpha}$, $\operatorname{var}(\widehat{\alpha})$, is obtained from the regression residuals 
through the heterocedasticity autoregressive consistent (HAC) procedure in Newey and West (1987), and the $M \times M$ variance-covariance matrix of age response function, $\operatorname{var}\left(\widehat{g_{\kappa}}\right)$, can be computed easily by the following

$$
\operatorname{var}\left(\widehat{g_{\kappa}}\right)=\Psi_{\kappa}^{\prime} \operatorname{var}(\widehat{\alpha}) \Psi_{\kappa}
$$

where $\widehat{g}_{\kappa}=\left(\widehat{g}_{\kappa}\left(\bar{s}_{1}\right), \cdots, \widehat{g}_{\kappa}\left(\bar{s}_{M}\right)\right)^{\prime}=\Psi_{\kappa}^{\prime} \widehat{\alpha}$ and $\Psi_{\kappa}=\left(\Psi\left(\bar{s}_{1}\right), \cdots, \Psi\left(\bar{s}_{M}\right)\right)$ is a $\kappa \times M$ matrix.

To choose the optimal $\kappa$, following the idea in Park (2010), by assuming that the dependent variable and regressors do not have stochastic trends, then we can use the $h$-block cross-validation (HCV) and the modified $h$-block CV (MHCV) criteria to choose the best $\kappa$ as proposed by Burman et al. (1994) and Racine (1997). For any given block size $h$, the HCV criterion is:

$$
\mathrm{HCV}=\frac{1}{T} \sum_{t=h}^{T-h}\left[x_{t}-z_{t}^{\prime} \widehat{\alpha}(t, h)\right]^{2}
$$

where $T$ is the number of original observations. The estimator $\widehat{\alpha}(t, h)$ is obtained by removing the $t$-th observation and $h$ observations before and after the $t$-th one in both sequances of $\left\{x_{t}\right\}$ and $\left\{z_{t}\right\}$. As suggested by the simulation study by Burman et al. (1994), $h$ can be simply taken to be $h=T / 6$. But the ratio $\kappa / T$ is not trivial. Therefore, it would be better to use the modified $h$-block CV criterion, which can be shown to have the following form

$$
\mathrm{MHCV}=\mathrm{HCV}+\frac{1}{T^{2}} \sum_{t=h}^{T-h} \sum_{s=1}^{T}\left[x_{s}-z_{s}^{\prime} \widehat{\alpha}(t, h)\right]^{2}+\frac{1}{T} \sum_{t=1}^{T}\left(x_{t}-z_{t}^{\prime} \widehat{\alpha}\right)^{2}
$$

Thus, the best $\kappa$ can be chosen by minimizing the above two CV criteria.

\subsection{Empirical Results From Single Equation Regressions}

We take the U.S. data to estimate the age impact functions for CPI inflation, threemonth Treasury yield, and the ex post real rate calculated by the three-month nominal yield subtracted with actual realized inflation. The annual demographic age distribution from the U.S. Census Bureau has been depicted in Figure 2. To match the quarterly frequency of the three macroeconomic series, we interpolate the annual distribution to 
obtain the quarterly distribution based on the assumption that the quarterly birth rate and quarterly death rate do not have seasonality within a year, so that we can compute the quarterly birth rate and death rate from their corresponding annual rate.

For a preliminary study conducted here, for each economic variable, we run the regression on the age distribution to obtain a related age impact function. With the selection criteria of MHCV, $\kappa$ is 5 for both inflation and nominal rate. Although the selected $\kappa$ is 2 for real rate from a single equation study, we choose it to be 5 in order to incorporate the economic restriction from the Fisher equation. Figure 3 presents the resulting impact functions.
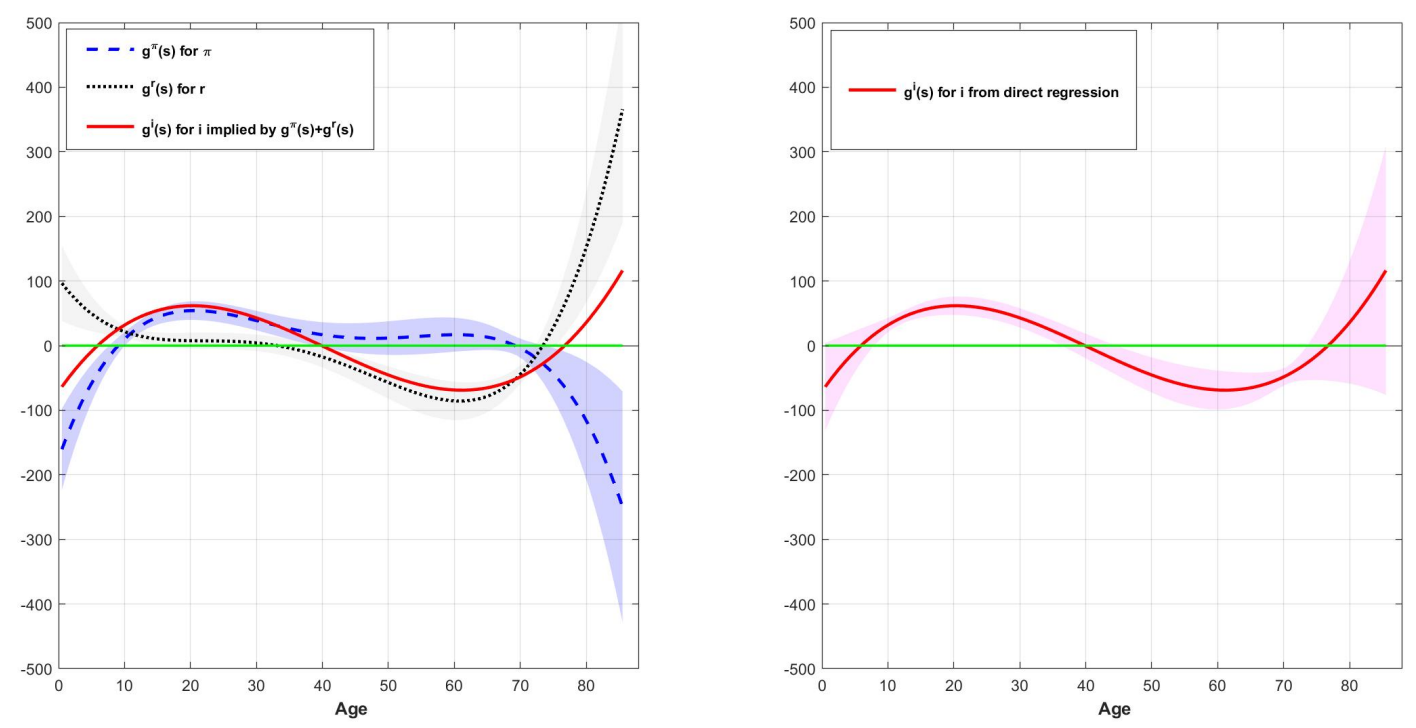

Figure 3. Impact functions from single equation regressions

From Figure 3 , the left panel shows the impact functions on inflation in dashed line, on real rate in dotted line, and the sum of the two functions in solid line to indicate the implied impact on nominal short rate. The function of real rate exhibits a $U$ shape, mirroring the inverted-U shape of impact function for savings found in Park et al. (2010), consistent with the life-cycle hypothesis. The impact function for inflation has an inverted U-shape, with different impacts from the young, middle, and old ages. The negative impact of the old-agers on inflation is consistent with the deflationary effect in a political-economic equilibrium (Katagiri et al., 2020). Although the U-shape function on real rate and the 
inverted U-shape function on inflation are largely to the opposite direction, they are not symmetric with respect to the zero line and their sum turns out to be S-shaped function on nominal short rate, indicated by the red solid line.

The right panel of Figure 3 plots the impact function estimated directly for nominal short rate. In comparison with the indirect impact shown on the left panel, the S-shaped pattern of the impact function on nominal rate is confirmed, being significantly positive for the young age between 10 and 35 and significantly negative for the middle to old age between 45 and a little above 73 .

According to (2.1), the time-varying trend can be computed as the integral of the age distribution and the impact function. With the estimation results of the impact functions, it amounts to computing the sum of the products of the discretized age density and its impact at each age group. Figure 4 plots the resulting trends for each variable. The left panel plots the trends for inflation in dashed line and real rate in dotted line, and the sum of them in solid line to indicate the implied trend of nominal rate. The right panel plots the trend for nominal short rate computed from the direct regression results, in contrasting with the actual nominal rate. A few observations are summarized as follows:

1. The demography-driven trend in inflation displays a downward tendency since mid 1970s and stabilizes since mid 1990s. The trend in real rate declines continuously since mid 1990s. These patterns are like those found in Bauer and Rudebusch (2020).

2. It is evident that the demography-driven trend of nominal rate traces the actual rate very well and smoothly.

3. The indirectly computed trend from the left panel is very consistent to the directly computed trend in the right panel.

These evidences from the single equation regressions strongly support the life-cycle hypothesis on a demography-driven trend in the nominal short rate, via population age impact on the real rate and inflation, respectively. But the estimation from single equation regression suffers from substantial parameter uncertainty with limited data. For example, 

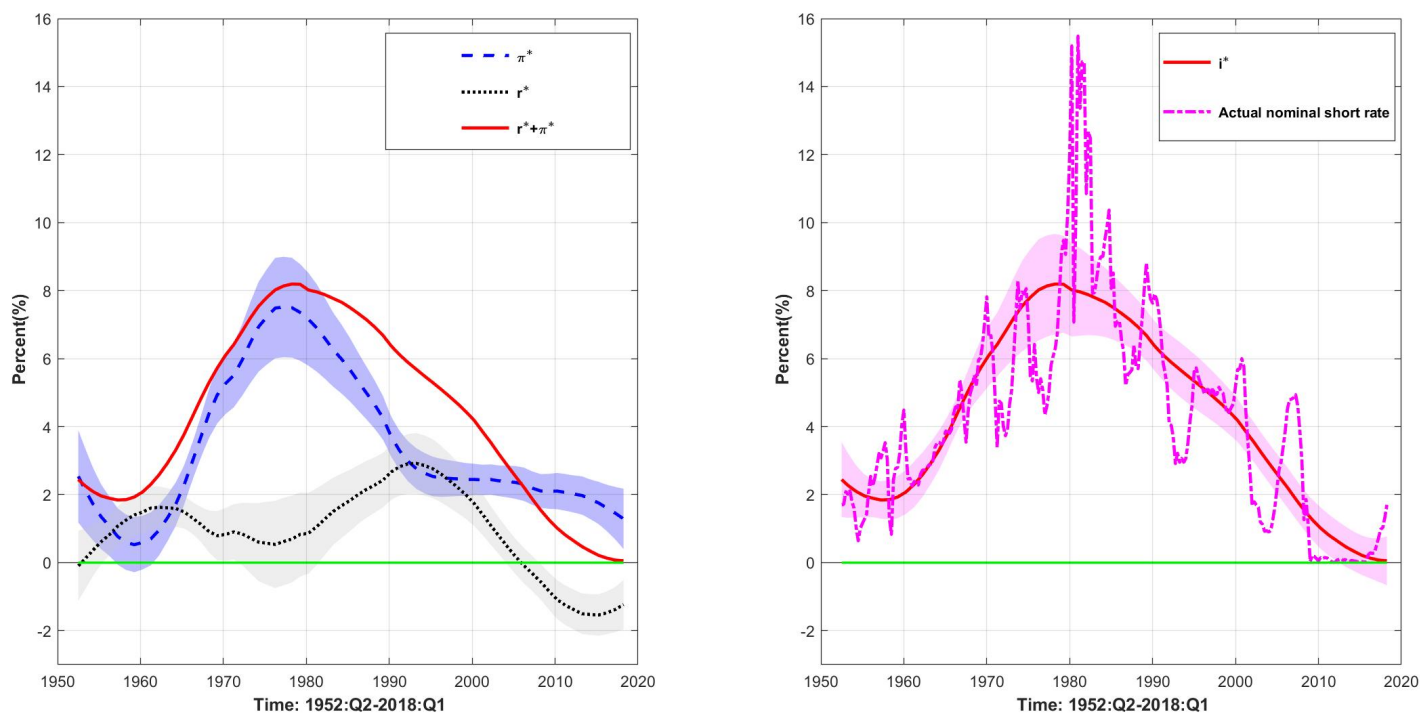

Figure 4. Demographic-driven trends from single equation regressions

the S-shaped age impact function on nominal short rate is not significant for the very young and old ages, which then affects uncertainty on the estimated trend. In the following section, we shall derive an affine arbitrage-free term structure model to fully explore the whole term structure to improve the inference of the age impact function on one hand, and utilize the demography-driven trend to explain the yield curve dynamics on the other hand.

\section{$3 \quad$ No-arbitrage Term Structure Model with Trending Fundamentals}

\subsection{Short Rate Equation}

First, denote the short rate $y_{t, 1}$ of the term structure as $i_{t}$,

$$
y_{t, 1}=i_{t}
$$

which is composed of a long-run trend, $i_{t}^{*}$, and a cyclical component, $\tilde{i}_{t}$,

$$
i_{t}=i_{t}^{*}+\tilde{\imath}_{t}
$$


and then, assume that the time-varying trend, $i_{t}^{*}$, is driven by the demographic age distribution through its impact $g(s)$ on the aggregated saving of each age cohort $s$,

$$
i_{t}^{*}=\int_{\underline{\mathrm{S}}}^{\bar{S}} f_{t}(s) g(s) d s,
$$

where the age span is $s \in[\underline{S}, \bar{S}], f_{t}(s)$ denotes the density function of demographic distribution at time $t$, and $g(s)$ represents age impact function to the short rate. Also, the cyclical component is driven by short- to medium-run cyclical factors $X_{t}$ with a typical affine representation

$$
\tilde{\imath}_{t}=\delta_{0}+\delta_{1} X_{t}
$$

Then, the short rate equation is summarized as

$$
y_{t, 1}=\underbrace{\int_{\underline{\mathrm{S}}}^{\bar{S}} f_{t}(s) g(s) d s}_{i_{t}^{*}}+\underbrace{\delta_{0}+\delta_{1} X_{t}}_{\tilde{\imath}_{t}} .
$$

\subsection{State Dynamics}

$f_{t}$ and $X_{t}$ denote both state factors, with the former being a slow-moving or long-term exogenous functional factor and the latter driving short- to medium-run fluctuations.

\subsubsection{Slow-Moving Functional State of Age Density}

We denote the total population at time $t$ as $L_{t}$, and the population of each age cohort on the age support $\left[s_{m-1}, s_{m}\right)$ as $L_{t}^{s_{m}}$, who were born in the time interval $\left(t-s_{m}, t-s_{m}+1\right]$. In practice, the age density can be approximated by the population weight of each age cohort in the $M$ age intervals, $\left[0, s_{1}\right),\left[s_{1}, s_{2}\right), \ldots,\left[s_{m-1}, s_{m}\right), \ldots,\left[s_{M-1}, \bar{S}\right]$,

$$
w_{t}\left(s_{m}\right)=\int_{s_{m-1}}^{s_{m}} f_{t}(s) d s \approx L_{t}^{s_{m}} / L_{t} .
$$

Suppose birth rate, $b_{t}$, is exogenous, such that the newly born cohort from the interval $(t-1, t]$ is $L_{t}^{1}=b_{t} L_{t-1}$. Given a survival rate function $P_{t}^{s_{m}, s_{m+1}}$ for population cohort $L_{t}^{s_{m}}$, the survived number of this cohort in the next period is $L_{t+1}^{s_{m+1}}=L_{t}^{s_{m}} P_{t}^{s_{m}, s_{m+1}}$. It is assumed that the survival rate function is stable over a long span in normal times without 
disruption of wars or natural disasters, which can be treated approximately constant as $P^{s_{m}, s_{m+1}}$. These assumptions have two implications in practice. First, the survival rate can be approximated as a moving average over a past interval, e.g., a few years back. Second, the prediction of future population over the medium- to long-run, say 1-10 years, can be computed as

$$
E_{t}\left(L_{t+h}^{s_{m+h}}\right)=L_{t}^{s_{m}} \prod_{j=1}^{h} P_{t+j-1}^{s_{m+j}, s_{m+j}}
$$

which should be relatively precise, given approximately constant $P^{s_{m+j-1}, s_{m+j}}$. Then, given reasonable projection on the birth rate, the age density of the short- to medium-horizon can be predicted with little uncertainty using the projected population weight

$$
E_{t}\left[w_{t+h}\left(s_{m+h}\right)\right]=E_{t} \int_{s_{m+h-1}}^{s_{m+h}} f_{t+h}(s) d s=E_{t}\left[L_{t+h}^{s_{m+h}} / L_{t+h}\right]
$$

where $E_{t} L_{t+h}=E_{t}\left(\sum_{m=1}^{M} L_{t+h}^{s_{m}}\right)$.

\subsubsection{Business Cycle Factors}

As suggested by Diebold and Li (2006), the state dynamics of the cyclical mean-reverting process under the physical measure can be modeled by a vector autoregression (VAR) of order $1, \operatorname{VAR}(1)$

$$
X_{t}=\mu+\Phi X_{t-1}+v_{t}
$$

where $v_{t} \sim N(0, \Omega)$, and state dynamics under the risk-neutral measure can be characterized by $\operatorname{VAR}(1)$ too as

$$
X_{t}=\mu^{Q}+\Phi^{Q} X_{t-1}+v_{t}^{Q}
$$

where $v_{t}^{Q} \sim N(0, \Omega)$.

\subsection{Interest Rate Term Structure}

A general form of yields also contains the two types of components of an affine form,

$$
y_{t, n}=y_{t, n}^{*}+\tilde{y}_{t, n}=\int_{\underline{\mathbf{S}}}^{\bar{S}} f_{t, n}(s) g(s) d s+a_{n}+b_{n}^{\prime} X_{t},
$$


where each yield of maturity is composed of a time-varying long-term trend driven by the demographic age distribution, and a cyclical component $\tilde{y}_{t, n}$. Next, it will show that expectation hypothesis and no-arbitrage argument impose cross-equation restrictions on the functional factors $f_{t, n}(s)$, and factor loadings $a_{n}$ and $b_{n}$ based on the short rate setting and state dynamics.

First, expectation hypothesis on the slow-moving demography-driven trend $y_{t, n}^{*}$ : Since the demographic distribution is slow moving and can be well predicted with little uncertainty in normal times, the expectation hypothesis is likely to hold that $y_{t, n}^{*}$ is an expected average of the long-run component of future short rate $i_{t+h}^{*}$, which is driven by the future demographic distribution $f_{t+h}$, that is $i_{t+h}^{*}=\int_{\underline{\mathrm{S}}}^{\bar{S}} f_{t+h}(s) g(s) d s$, with $h=0, \ldots, n-1$.

$$
\begin{aligned}
y_{t, n}^{*} & =\underbrace{\frac{1}{n} E_{t}\left[\sum_{h=0}^{n-1} i_{t+h}^{*}\right]}_{\text {E.H. }}=\frac{1}{n} E_{t}\left[\sum_{h=0}^{n-1} \int_{\underline{\underline{S}}}^{\bar{S}} f_{t+h}(s) g(s) d s\right]=\int_{\underline{\mathbf{S}}}^{\bar{S}}\left[\frac{1}{n} \sum_{h=0}^{n-1} E_{t}\left\{f_{t+h}(s)\right\}\right] g(s) d s \\
& =\int_{\underline{S}}^{\bar{S}} f_{t, n}(s) g(s) d s
\end{aligned}
$$

with

$$
f_{t, n}(s)=\frac{1}{n} \sum_{h=0}^{n-1} E_{t}\left[f_{t+h}(s)\right],
$$

where $f_{t, 1}(s) \equiv f_{t}(s)$ and $f_{t+h}(s)$ can be approximated by the predicted population weights as in (3.3). The implication is clear that the slow-moving demographic age distribution in the long term yields, $f_{t, n}(s)$, is an average of the expected demographic age distribution evolving through the holding horizon.

Second, no-arbitrage yield cyclical component $\tilde{y}_{t, n}$ with state dynamics given in (3.4) and (3.5) is written as

$$
\tilde{y}_{t, n}=a_{n}+b_{n}^{\prime} X_{t}
$$

where, starting from $a_{1}=\delta_{0}$ and $b_{1}=\delta_{1}$ with short rate parameters as in (3.1), $a_{n}=-A_{n} / n$ and $b_{n}=-B_{n} / n$ can be derived from the partial differential equations (PDEs) in following forms (Ang and Piazzesi, 2003),

$$
B_{n+1}^{\prime}=B_{n}^{\prime} \Phi^{Q}+B_{1}^{\prime},
$$


and

$$
A_{n+1}=A_{n}+B_{n}^{\prime} \mu^{Q}+\frac{1}{2} B_{n}^{\prime} \Omega B_{n}+A_{1} .
$$

\subsection{Econometric Representation}

\subsubsection{General Representation}

To summarize, the econometric representation of the generalized affine term structure model is

$$
y_{t, n}=\int_{\underline{S}}^{\bar{S}} f_{t, n}(s) g(s) d s+a_{n}+b_{n}^{\prime} X_{t}+\epsilon_{t, n}, \quad \epsilon_{t, n} \sim N\left(0, \sigma_{\epsilon}^{2}\right),
$$

termed as semiparametric affine term structure model (SP-ATSM), where $X_{t}$ follows a $\operatorname{VAR}(1)$ model as

$$
X_{t}=\mu+\Phi X_{t-1}+v_{t}, \quad v_{t} \sim N(0, \Omega)
$$

with $f_{t, n}(s)$ defined in (3.7), and $a_{n}=-A_{n} / n$ and $b_{n}=-B_{n} / n$ defined in the PDEs in (3.9) and (3.8), respectively.

It can be seen that the yield is an affine form of the factors $f_{t}$ and $X_{t}$ with crossequation restrictions under the EH and no-arbitrage theory. The slow-moving component is forward-looking, with the current and future demographic age structure exerting impact through the age impact function $g(s)$.

\subsubsection{Specific Representation on the Cyclical Factors}

The stationary no-arbitrage autoregressive part of yields, $\tilde{y}_{t, n}$, can be described by any typical affine term structure model (ATSM) with specified factors of $X_{t}$, such as latent factors or observed macroeconomic factors. To keep parsimony in our semiparametric set up, we shall choose a simple affine arbitrage-free Nelson-Siegel (AFNS) model with the NS factor interpolation

$$
B_{n}=\left(-n,-\frac{1-e^{-\lambda n}}{\lambda}, n e^{-\lambda n}-\frac{1-e^{-\lambda n}}{\lambda}\right)^{\prime},
$$


which corresponds to a risk neutral transition matrix taking the specific form

$$
\Phi^{Q}=\left(\begin{array}{ccc}
1 & 0 & 0 \\
0 & e^{-\lambda} & \lambda e^{-\lambda} \\
0 & 0 & e^{-\lambda}
\end{array}\right)
$$

as shown in Christensen et al. (2011) for a continuous-time setting and Hong et al. (2019) for a discrete-time case. One can then impose the restriction that $a_{1}=-A_{1}=\delta_{0}=0$ for normalization. For parameter identification, we restrict $\mu^{Q}=\left(\mu_{L}^{Q}, 0,0\right)^{\prime}$ like in Hong et al. (2019). Conditional on this basic AFNS model, one has an ability to make a comparison between our demographic trend driven semiparametric AFNS (SP-AFNS) model in (3.10), the pure AFNS model, and an extended AFNS augmented with single demographic index of MY ratio.

\section{Estimation Method}

In this section, we first introduce how the two components and related parameters can be estimated in the classical framework, if they could be separately observed. Then, we discuss the joint estimation strategy within a Bayesian framework.

\subsection{Nonparametric Trend Extraction Under EH}

In Section 2, we estimate the age impact function of nominal short rate on the demographic age distribution using a linear estimator in a single equation. In this section, we exploit the rich information in the term structure of interest rates to robustly infer the common age impact function $g(s)$ under the EH from the structural equation of yields in (3.6). Similar to single equation regressions, we use the aforementioned Fourier flexible series approach to approximate the functional form as in (2.2), then, the resulting approximation is

$$
y_{t, n}^{*}=\int_{\underline{\mathrm{S}}}^{\bar{S}} f_{t, n}(s) g(s) d s \approx \sum_{i=1}^{\kappa} \alpha_{i} \int_{\underline{\mathrm{S}}}^{\bar{S}} f_{t, n}(s) \psi_{i}(s) d s
$$

which is similar to (2.3), except that we impose the EH restriction on demographic distribution as shown in (3.7). Again, on the discretized age support with weights of each

cohort at time $t$ and $t+h$, as shown in (3.2) and (3.3), then, the integral in (4.1) can be 
denoted as $z_{t, n, i}$ and approximated as

$$
z_{t, n, i} \equiv \int_{0}^{\bar{S}} f_{t, n}(s) \psi_{i}(s) d s \approx \frac{1}{n} \sum_{h=0}^{n-1} E_{t}\left[\sum_{m=1}^{M} w_{t+h}\left(s_{m}\right) \psi_{i}\left(\bar{s}_{m}\right)\right]
$$

with $\bar{s}_{m}=\left(s_{m-1}+s_{m}\right) / 2$.

Given the above approximation, (4.1) can also be written as a linear form,

$$
y_{t, n}^{*}=\alpha^{\prime} z_{t, n}
$$

where $\alpha$ is defined in (2.5) and similar to $(2.5), z_{t, n}=\left(z_{t, n, 1} \cdots, z_{t, n, \kappa}\right)^{\prime}$ with $z_{t, n, i}$ defined as in (4.2). For different yields to maturity, we have the common parameter vector $\alpha$, so that a simultaneous equations regression is formed as

$$
Y_{t}^{*}=Z_{t}^{\prime} \alpha
$$

where $Y_{t}^{*}$ is an $N \times 1$ vector of $N$ observed yields and $Z_{t}=\left(z_{t, n_{1}}^{\prime}, \cdots, z_{t, n_{N}}^{\prime}\right)$ is a $\kappa \times N$ matrix. Then, with a sample of $T$ observations for each maturity of yields, a compact linear form can be written as

$$
Y^{*}=Z \alpha
$$

where $Y^{*}=\left(Y_{1}^{*^{\prime}}, \cdots, Y_{T}^{*^{\prime}}\right)^{\prime}$ is an $N T \times 1$ vector and $Z=\left(Z_{1}, Z_{2}, \cdots, Z_{T}\right)^{\prime}$ is an $N T \times \kappa$ matrix. Then, the OLS estimator of $\alpha$ is

$$
\widehat{\alpha}=\left(Z^{\prime} Z\right)^{-1} Z^{\prime} Y
$$

with $Y=\left(Y_{1}^{\prime}, \cdots, Y_{T}^{\prime}\right)^{\prime}$ containing actual data of yields.

Once $\widehat{\alpha}$ is obtained, then, its variance and covariance matrix can be estimated through the HAC procedure in Newey and West (1987) from the above simultaneous equations regression. Thus, one can obtain the age impact function and its variance-covariance matrix, similar to (2.6) and (2.7) as follows, for each $1 \leq m \leq M$,

$$
\widehat{g}_{\kappa}\left(\bar{s}_{m}\right)=\widehat{\alpha}^{\prime} \Psi\left(\bar{s}_{m}\right) \quad \text { and } \quad \operatorname{var}\left(\widehat{g_{\kappa}}\right)=\Psi_{\kappa}^{\prime} \operatorname{var}(\widehat{\alpha}) \Psi_{\kappa}
$$

where $\Psi_{\kappa}$ is defined in (2.7). 
Again, we use the h-block CV and the modified h-block CV criteria to choose the best $\kappa$ similar to the idea described in Section 2.2. For any given block size $h$, the $h$-block CV criterion for the structural estimation is given by

$$
\mathrm{HCV}=\frac{1}{T} \sum_{t=h}^{T-h}\left\|Y_{t}, Z_{t}^{\prime} \widehat{\alpha}(t, h)\right\|,
$$

where $\|A, B\|$ denotes the Euclidean distance between the corresponding elements in the vectors $A$ and $B$, and the estimator $\widehat{\alpha}(t, h)$ is obtained by removing the $t$-th observation and $h$-observations before and after the $t$-th in both $Z$ and $Y$. Similarly, $h=T / 6$ is set as in Section 2.2. The modified $h$-block CV criterion has the following form

$$
\mathrm{MHCV}=\mathrm{HCV}+\frac{1}{T} \sum_{t=h}^{T-h} \sum_{s=1}^{T}\left\|Y_{s}, Z_{s}^{\prime} \widehat{\alpha}(t, h)\right\|+\frac{1}{T} \sum_{t=1}^{T}\left\|Y_{t}, Z_{t}^{\prime} \widehat{\alpha}\right\| .
$$

Then, the best $\kappa$ can be chosen by minimizing the above two CV criteria.

\subsection{Parametric Estimation on the Cyclical Component}

Given observable $\tilde{y}_{t, n}$, the ATSM model has a state space representation

$$
\tilde{y}_{t, n}=a_{n}+b_{n}^{\prime} X_{t}+\epsilon_{t, n}, \quad \epsilon_{t, n} \sim N\left(0, \sigma_{\epsilon}^{2}\right),
$$

and

$$
X_{t}=\mu+\Phi X_{t-1}+v_{t}, \quad v_{t} \sim N(0, \Omega),
$$

where $a_{n}$ and $b_{n}$ are nonlinear functions of the underlying parameters, as shown in (3.9) and (3.8). Based on the distributional assumptions on the error terms, the Gaussian model can be estimated by maximum likelihood estimation (MLE) with Kalman filter (Ang and Piazzesi, 2003; Christensen et al., 2011). Nonlinearity in the factor loadings of measurement equations makes the MLE challenging both on the searching for global optimum and on learning parameter uncertainties. To overcome this difficulty, we propose to use the MCMC method, which has gained popularity in estimating such models, especially, when hidden states are involved (Ang et al., 2011). 


\subsection{Joint Semiparametric Estimation Using Bayesian Method}

A simple way to estimate the model jointly can be an iterated estimation with the above two steps until certain criteria is reached. In the initial step, it is assumed that the yields are dominated by the long-term component with mean-zero cyclical component. Conditional on the extracted long-term part $y_{t, n}^{*}$, the rest are modeled as $\tilde{y}_{t, n}$. However, consistency is not guaranteed in this procedure. In the first place, the simultaneous zero assumption on the cyclical components in the simultaneous equations is not compatible with the no-arbitrage restriction. Therefore, we choose to jointly estimate the model with the MCMC approach, described next.

The parameters to be estimated are $\Theta=\left\{\alpha, \sigma_{\epsilon}^{2}, \mu, \Phi, \Omega, \mu_{L}^{Q}, \lambda\right\}$, and the latent variables to be estimated are $X=\left\{X_{1}, \cdots, X_{T}\right\}$. For ease of illustration, the parameters are divided into four blocks as: $\Theta_{1}=\left\{\alpha, \sigma_{\epsilon}^{2}\right\}, \Theta_{2}=\{\mu, \Phi\}, \Theta_{3}=\{\Omega\}$, and $\Theta_{4}=\left\{\mu_{L}^{Q}, \lambda\right\}$. The Metropolis-Hastings (MH) within Gibbs sampling algorithm is used to do the Bayesian estimation closely related to Ang et al. (2007) and Hong et al. (2019). The detailed procedures of drawing the latent states and blocks of parameters are as follows.

\subsubsection{Drawing Latent Variables $X_{t}$}

Given the observed yield data $Y=\left(Y_{1}, \cdots, Y_{T}\right)$, the transformed linear regressors $Z=$ $\left(Z_{1}, \cdots, Z_{T}\right)$, and the parameters drawn from the last step, $\Theta^{d-1}$, the forward filtering and backward smoothing algorithm as in Carter and Kohn (1994) is employed to draw the latent variables involved in $X$, denoted as $X^{d}$ in the $d$-th draw.

\subsubsection{Drawing $\mu$ and $\Phi$}

Given $X^{d}$, as $X_{t}$ follows a VAR process in (3.4), then the draw of $\mu$ and $\Phi$ can be a standard Gibbs sampling with conjugate normal priors and posteriors. Note that the 
posterior of $\mu$ and $\Phi$ conditional on $Y, Z, X^{d}$, and the other parameters is:

$$
\begin{aligned}
& P\left(\mu, \Phi \mid \Theta_{1}^{d-1}, \Theta_{3}^{d-1}, \Theta_{4}^{d-1}, Y, Z, X^{d}\right) \\
& \propto P\left(Y \mid \Theta_{1}^{d-1}, \Theta_{3}^{d-1}, \Theta_{4}^{d-1}, Z, X^{d}\right) P\left(X^{d} \mid \mu, \Phi, \Omega^{d-1}\right) P\left(\mu_{0}, \Phi_{0}\right) \\
& \propto P\left(X^{d} \mid \mu, \Phi, \Omega^{d-1}\right),
\end{aligned}
$$

where $P\left(X^{d} \mid \mu, \Phi, \Omega^{d-1}\right)$ is the likelihood function, which is normally distributed based on (3.4), and $P\left(\mu_{0}, \Phi_{0}\right)$ is the prior of $\mu$ and $\Phi$. The validity of going from the first line to

the second line is ensured by the PDEs in (3.9) and (3.8): given $\Theta_{4}=\left\{\mu_{L}^{Q}, \lambda\right\}$, the bond price is independent of $\mu$ and $\Phi$. Here, we use the non-informative prior (the so-called Jeffreys prior) over $\mu$ and $\Phi$, thus we have the likelihood function left, which can be drawn from normal distribution. We denote them as $\mu^{d}$ and $\Phi^{d}$, respectively.

\subsubsection{Drawing $\Omega$}

Since $\Omega$ appears in the PDEs of (3.9) in a nonlinear way, there is not an analytical expression of conditional posterior distribution for $\Omega$. We note that the posterior of $\Omega$ conditional on $Y, Z$, and $X^{d}$ as well as the other parameters is:

$$
\begin{aligned}
& P\left(\Omega \mid \Theta_{1}^{d-1}, \Theta_{2}^{d}, \Theta_{4}^{d-1}, Y, Z, X^{d}\right) \\
& \propto P\left(Y \mid \Theta_{1}^{d-1}, \Theta_{2}^{d}, \Theta_{4}^{d-1}, \Omega, Z, X^{d}\right) P\left(X^{d} \mid \mu^{d}, \Phi^{d}, \Omega\right) P\left(\Omega_{0}\right),
\end{aligned}
$$

where $P\left(\Omega_{0}\right)$ is the prior of $\Omega$, which suggests an independent Metropolis draw. We draw candidate $\Omega$ from the proposal density $q(\Omega)=P\left(X^{d} \mid \mu^{d}, \Phi^{d}, \Omega\right) P\left(\Omega_{0}\right)$, which is an inverse Wishart (IW) distribution if the prior $P\left(\Omega_{0}\right)$ is specified to be IW, so that $q(\Omega)$ is an IW natural conjugate. The proposal draw of $\Omega$ for the $d$-th draw is then accepted with probability $\beta$ as:

$$
\begin{aligned}
\beta & =\min \left\{\frac{P\left(\Omega^{d} \mid \Theta_{1}^{d-1}, \Theta_{2}^{d}, \Theta_{4}^{d-1}, Y, Z, X^{d}\right)}{P\left(\Omega^{d-1} \mid \Theta_{1}^{d-1}, \Theta_{2}^{d}, \Theta_{4}^{d-1}, Y, Z, X^{d}\right)} \frac{q\left(\Omega^{d-1}\right)}{q\left(\Omega^{d}\right)}, 1\right\} \\
& =\min \left\{\frac{P\left(Y \mid \Omega^{d}, \Theta_{1}^{d-1}, \Theta_{2}^{d}, \Theta_{4}^{d-1}, Z, X^{d}\right)}{P\left(Y \mid \Omega^{d-1}, \Theta_{1}^{d-1}, \Theta_{2}^{d}, \Theta_{4}^{d-1}, Z, X^{d}\right)}, 1\right\}
\end{aligned}
$$


where the numerator and denominator of the last line are the likelihood functions, which are normally distributed from the assumption of normality for the measurement error $\epsilon_{t, n}$. Therefore, $\beta$ is the likelihood ratio of the $d$-th draw relative to that of the $(d-1)$-th draw.

\subsubsection{Drawing $\lambda$ and $\mu_{L}^{Q}$}

Because both $\lambda$ and $\mu_{L}^{Q}$ appear in the measurement equations in nonlinear forms through the PDEs in (3.9) and (3.8), there are not analytical conditional posterior distribution expressions for these parameters. We draw $\lambda$ and $\mu_{L}^{Q}$ one by one using a MH algorithm. We illustrate the sampling of $\lambda$ as follows, and the sampling for $\mu_{L}^{Q}$ is similar. The posterior distributions of $\lambda$ conditional on $Y, Z, X^{d}$, and the other parameters are:

$$
\begin{aligned}
& P\left(\lambda \mid \Theta_{1}^{d-1}, \Theta_{2}^{d}, \Theta_{3}^{d}, \mu_{L}^{Q^{d-1}}, Y, Z, X^{d}\right) \\
& \propto P\left(Y \mid \Theta_{1}^{d-1}, \Theta_{2}^{d}, \Theta_{3}^{d}, \mu_{L}^{Q^{d-1}}, \lambda, Y, Z, X^{d}\right) P\left(\lambda_{0}\right) \\
& \propto P\left(Y \mid \Theta_{1}^{d-1}, \Theta_{2}^{d}, \Theta_{3}^{d}, \mu_{L}^{Q^{d-1}}, \lambda, Y, Z, X^{d}\right)
\end{aligned}
$$

where $P\left(\lambda_{0}\right)$ is the prior of $\lambda$. We use the non-informative prior, so that we have the likelihood function left. $\lambda$ enters the likelihood in a nonlinear way and we draw $\lambda$ using a random walk chain $\mathrm{MH}$ algorithm:

$$
\lambda^{d}=\lambda^{d-1}+\tau_{\lambda} \eta
$$

where $\eta \sim N(0,1)$ and $\tau_{\lambda}$ is the scale parameter to control the acceptance ratio. The acceptance probability of $\lambda^{d}$ is:

$$
\begin{aligned}
\beta & =\min \left\{\frac{P\left(\lambda^{d} \mid \Theta_{1}^{d-1}, \Theta_{2}^{d}, \Theta_{3}^{d}, \mu_{L}^{Q^{d-1}}, Y, Z, X^{d}\right)}{P\left(\lambda^{d-1} \mid \Theta_{1}^{d-1}, \Theta_{2}^{d}, \Theta_{3}^{d}, \mu_{L}^{Q^{d-1}}, Y, Z, X^{d}\right)} \frac{q\left(\lambda^{d-1} \mid \lambda^{d}\right)}{q\left(\lambda^{d} \mid \lambda^{d-1}\right)}, 1\right\} \\
& =\min \left\{\frac{P\left(\lambda^{d} \mid \Theta_{1}^{d-1}, \Theta_{2}^{d}, \Theta_{3}^{d}, \mu_{L}^{Q^{d-1}}, Y, Z, X^{d}\right)}{P\left(\lambda^{d-1} \mid \Theta_{1}^{d-1}, \Theta_{2}^{d}, \Theta_{3}^{d}, \mu_{L}^{Q^{d-1}}, Y, Z, X^{d}\right)}, 1\right\} \\
& =\min \left\{\frac{P\left(Y \mid \Theta_{1}^{d-1}, \Theta_{2}^{d}, \Theta_{3}^{d}, \mu_{L}^{Q^{d-1}}, \lambda^{d}, Y, Z, X^{d}\right)}{P\left(Y \mid \Theta_{1}^{d-1}, \Theta_{2}^{d}, \Theta_{3}^{d}, \mu_{L}^{Q^{d-1}}, \lambda^{d-1}, Y, Z, X^{d}\right)}, 1\right\} .
\end{aligned}
$$




\subsubsection{Drawing $\alpha$ and $\sigma_{\epsilon}^{2}$}

Conditional on the $d$-th draw of parameters in $\Theta_{2}^{d}, \Theta_{3}^{d}, \Theta_{4}^{d}$, latent variables $X^{d}$, observed yield data $Y$, and the transformed linear regressors $Z$, the measurement equation is reduced to a linear regression model. Thus, the conditional posterior of $\Theta_{1}$ can be drawn by the Gibbs sampling procedure with the normal distribution prior and inverse-Gamma distribution prior for $\alpha$, and $\sigma_{\epsilon}^{2}$, respectively; see, for example, Chapter 8 of Koop (2003) for details. In practice, we set the variance-covariance matrix of normal distribution prior for $\alpha$ to be the variance-covariance of the coefficients of the simultaneous equations regression, and let the prior mean be the OLS estimator from the simultaneous equations regression.

\section{$5 \quad$ Empirical Results}

We run the MCMC procedure as explained above for 220,000 draws and keep the last 200,000 draws after the initial 20,000 burn-ins. According to the single equation and simultaneous equations analysis, we have chosen $\kappa=5$. In what follows, we report the estimation results and discuss its inference and forecast comparisons.

\subsection{Parameter Estimation}

Table 1 reports the parameter estimation results. The upper part includes the parameters in the measurement equation. $\alpha$ for the impact function of demographic distribution driving trend are significantly estimated. The risk-neutral parameter $\lambda$ implies that the curvature factor reaches a peak loading around the maturity of 6 quarters, which is of shorter maturity than that in a traditional AFNS model with a higher value of $\lambda$. The lower part reports the state dynamic parameters of the NS factors in the physical VAR process. The diagonal elements in the autoregressive matrix are less persistent than the traditional estimates from an AFNS model without controlling the slow-moving trend.

Convergence diagnostics based on trace plots reported in Figure 5 and inefficiency factors in (Table 2) indicate convergence, though the inefficiency factors for parameters

in the measurement equation tend to be larger. The risk-neutral parameter $\mu_{L}^{Q}$ is the 
Table 1. Parameter estimates with MCMC method

\begin{tabular}{|c|c|c|c|c|}
\hline \multicolumn{5}{|c|}{ Measurement Equations } \\
\hline \multicolumn{5}{|l|}{ Trend } \\
\hline$\alpha_{1}$ & $\alpha_{2}$ & $\alpha_{3}$ & $\alpha_{4}$ & $\alpha_{5}$ \\
\hline-6.726 & 38.134 & -5.402 & 6.513 & -34.858 \\
\hline$(-32.294,17.850)$ & $(26.146,49.491)$ & $(-18.774,8.521)$ & $(-18.084,32.103)$ & $(-45.951,-23.144)$ \\
\hline \multicolumn{5}{|l|}{ Cycle } \\
\hline & $\lambda$ & $\mu_{l}^{Q} * 10^{4}$ & $\sigma_{\epsilon} * 4 * 10^{4}$ & \\
\hline & 0.283 & 2.998 & 8.276 & \\
\hline & $(0.269,0.295)$ & $(2.735,3.326)$ & $(8.013,8.557)$ & \\
\hline \multicolumn{5}{|c|}{ State Equations } \\
\hline$\Phi$ & $L_{t-1}$ & $S_{t-1}$ & $C_{t-1}$ & $\mu$ \\
\hline \multirow{2}{*}{$L_{t}$} & 0.964 & 0.087 & -0.053 & 0.0002 \\
\hline & $(0.925,1.003)$ & $(0.045,0.129)$ & $(-0.078,-0.027)$ & $(-0.0000,0.0004)$ \\
\hline \multirow{2}{*}{$S_{t}$} & -0.084 & 0.845 & 0.024 & -0.0003 \\
\hline & $(-0.143,-0.025)$ & $(0.783,0.907)$ & $(-0.014,0.061)$ & $(-0.0006,-0.0000)$ \\
\hline \multirow{2}{*}{$C_{t}$} & 0.031 & 0.423 & 0.591 & 0.0018 \\
\hline & $(-0.105,0.169)$ & $(0.282,0.566)$ & $(0.503,0.677)$ & $(0.0012,0.0025)$ \\
\hline \multirow[t]{5}{*}{$\Omega * 10^{4}$} & $\begin{array}{c}\mathbf{0 . 0 1 8} \\
(0.015,0.021)\end{array}$ & & & \\
\hline & -0.011 & 0.041 & & \\
\hline & $(-0.015,-0.008)$ & $(0.035,0.048)$ & & \\
\hline & -0.007 & 0.009 & 0.211 & \\
\hline & $(-0.015,0.001)$ & $(-0.001,0.021)$ & $(0.179,0.251)$ & \\
\hline
\end{tabular}

Note: Median of parameters drawn, and $90 \%$ confidence interval in the parenthesis consisting of $5 \%$ and $95 \%$ quantile of parameters drawn.

slowest to converge due to its nonlinearity in entering the measurement equations and the inference is much more difficult. However, with a large number of 200,000 posterior draws, the convergence has been achieved, as can be seen from the trace plots. 

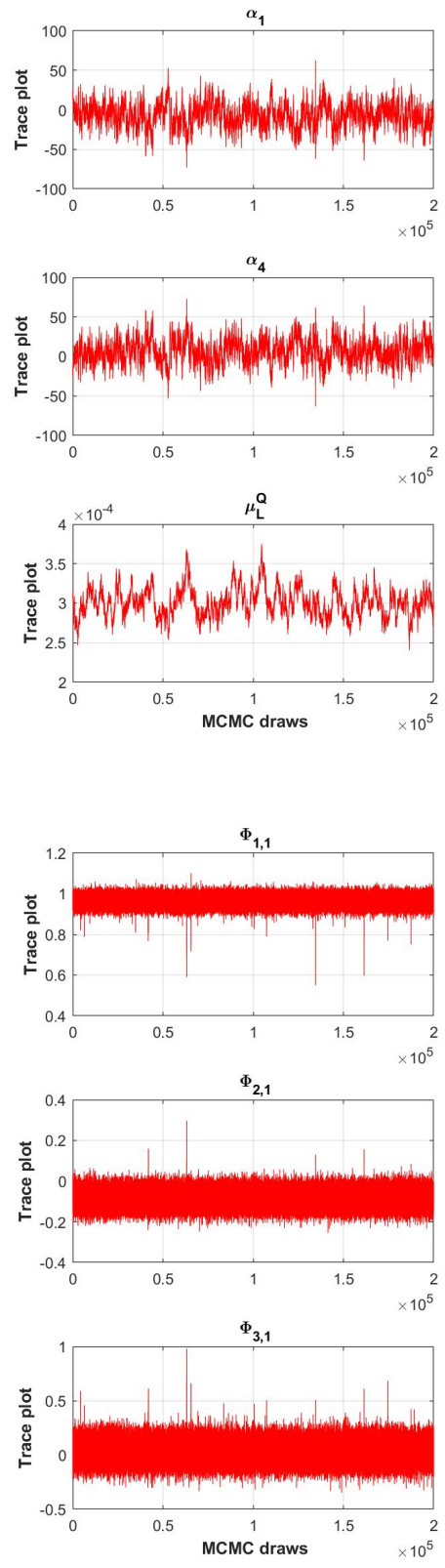

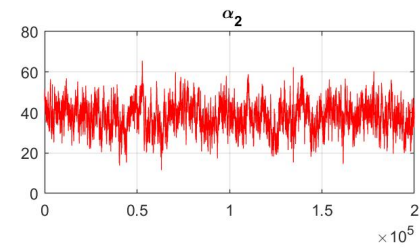

$\alpha_{5}$
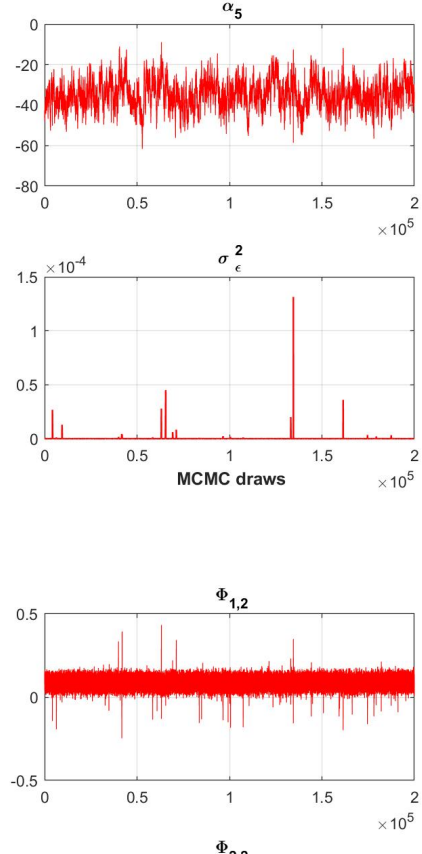

$\Phi_{2,2}$

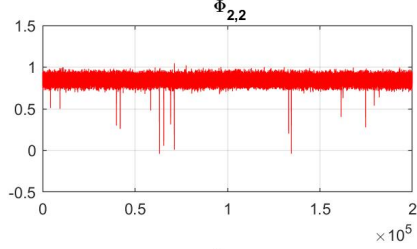

3,2

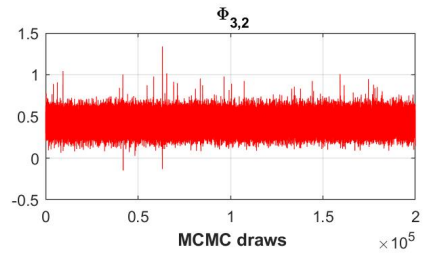

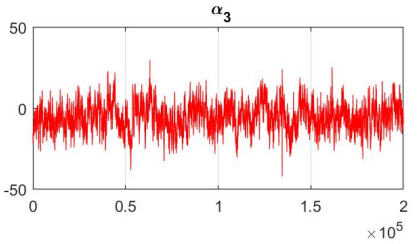
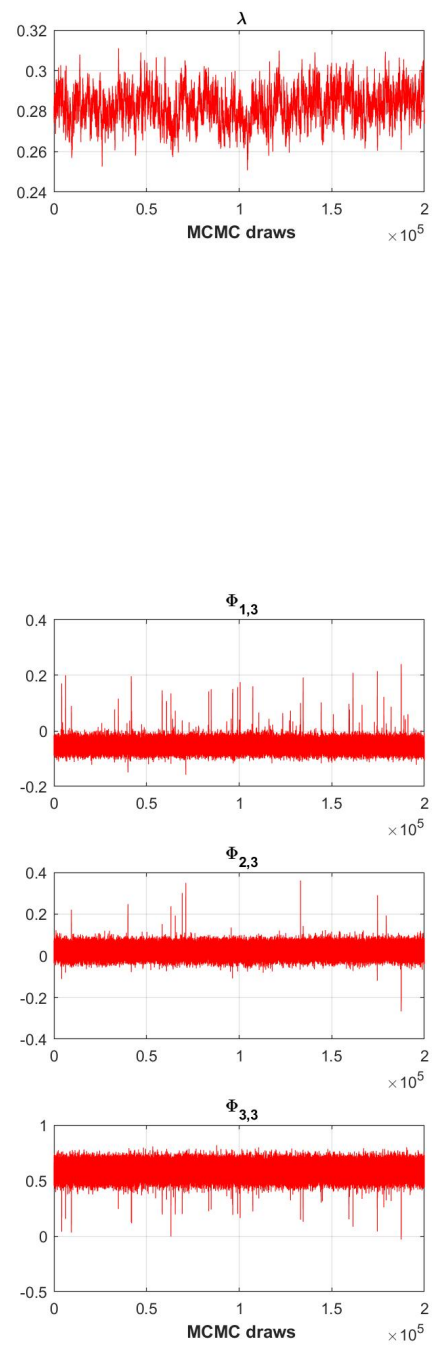

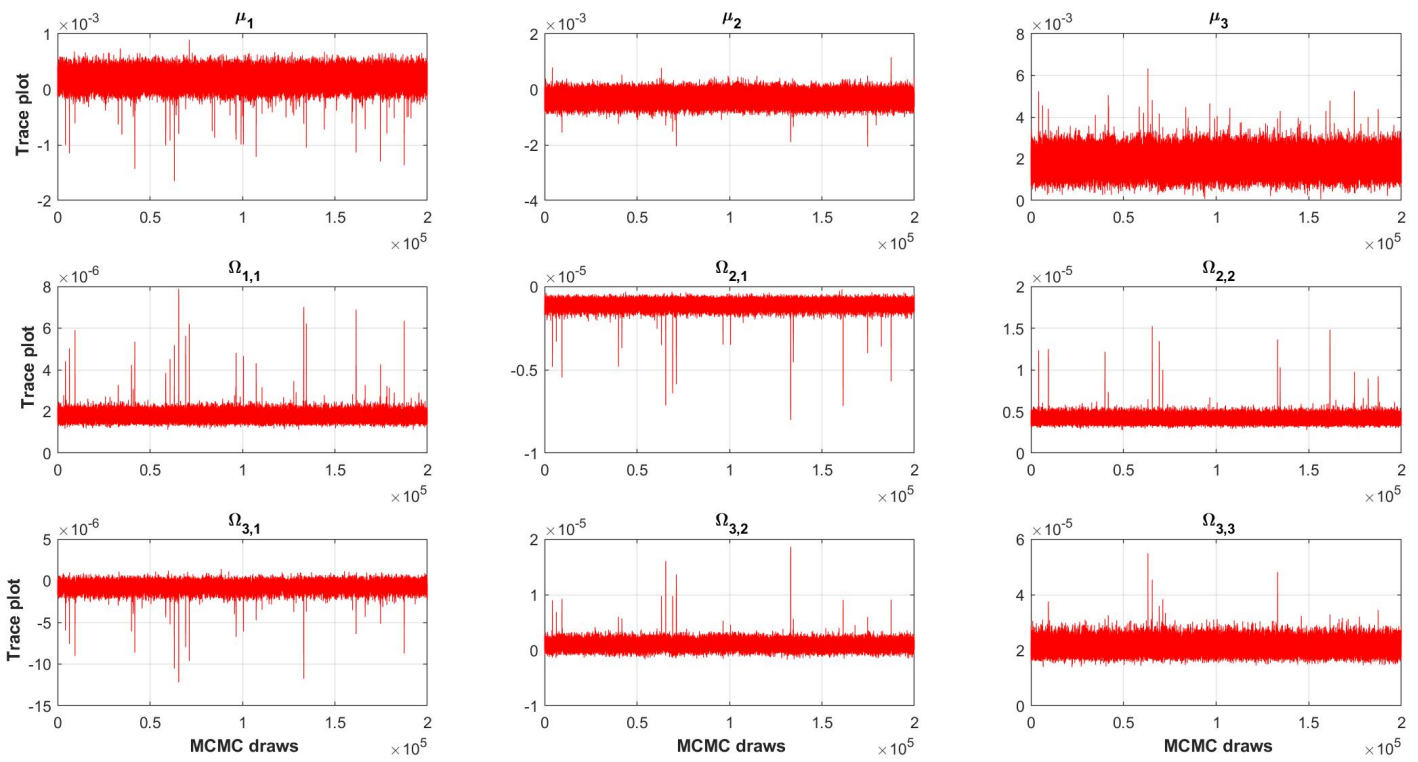

Figure 5. Trace plots of the posterior draws

Table 2. Inefficiency factor of parameter posterior draws

\begin{tabular}{|c|c|c|c|c|}
\hline \multicolumn{5}{|c|}{ Measurement Equations } \\
\hline \multicolumn{5}{|l|}{ Trend } \\
\hline$\alpha_{1}$ & $\alpha_{2}$ & $\alpha_{3}$ & $\alpha_{4}$ & $\alpha_{5}$ \\
\hline 322.492 & 323.999 & 322.524 & 322.476 & 324.202 \\
\hline \multicolumn{5}{|l|}{ Cycle } \\
\hline & $\lambda$ & $\mu_{l}^{Q}$ & $\sigma_{\epsilon}$ & \\
\hline & 299.639 & 443.211 & 1.162 & \\
\hline \multicolumn{5}{|c|}{ State Equations } \\
\hline$\Phi$ & $L_{t-1}$ & $S_{t-1}$ & $C_{t-1}$ & $\mu$ \\
\hline$L_{t}$ & 3.159 & 12.496 & 9.014 & 17.074 \\
\hline$S_{t}$ & 1.618 & 8.998 & 11.153 & 9.944 \\
\hline$C_{t}$ & 4.941 & 2.856 & 2.943 & 11.257 \\
\hline \multirow[t]{3}{*}{$\Omega$} & 12.902 & & & \\
\hline & 8.702 & 6.044 & & \\
\hline & 31.869 & 19.424 & 10.113 & \\
\hline
\end{tabular}

Note: Inefficiency factor is defined as in Chib and Ergashev (2009): $1+2 \sum_{l=1}^{500}\left(1-\frac{l}{500}\right) \rho(l)$, where $\rho(l)$ the sample autocorrelation at lag $l$ from each MCMC sequence.

Based on the estimates, the goodness of fit of this model is shown in Table 3 , which reports the descriptive statistics of the residuals in yields. The standard deviations of 
errors are mostly within 7 bps, and the RMSE or MAE are on average only between 6 and 7 bps.

Table 3. In-sample fit of SP-AFNS: Residual statistics (bps)

\begin{tabular}{cccccccc}
\hline Maturity & Mean & Median & Max & Min & Std & RMSE & MAE \\
\hline 1Q & -0.528 & -0.692 & 20.336 & -14.028 & 3.845 & 3.874 & 2.726 \\
\hline 4Q & 2.117 & 2.251 & 42.981 & -29.173 & 9.313 & 9.533 & 7.013 \\
\hline 8Q & -3.109 & -2.809 & 20.982 & -28.902 & 6.260 & 6.979 & 5.256 \\
\hline 12Q & 0.546 & 0.026 & 22.313 & -18.720 & 6.916 & 6.924 & 5.374 \\
\hline 16Q & 2.268 & 1.847 & 42.438 & -24.265 & 6.379 & 6.759 & 4.688 \\
\hline 20Q & -1.299 & -1.127 & 19.070 & -31.525 & 6.938 & 7.046 & 5.339 \\
\hline Average & -0.001 & -0.084 & 28.020 & -24.435 & 6.608 & 6.852 & 5.066 \\
\hline
\end{tabular}

Note: This table shows the descriptive statistics of the median residuals in yields.

\subsection{Impact function and trend}

Figure 6 plots the estimated age impact function and implied trend from the joint model, where the solid lines are the median and the darker shaded area indicates the $95 \%$ confidence band. We also plot the corresponding single equation results using dashed lines with a lighter gray area indicating its $95 \%$ confidence interval. By comparison, with crossmaturity information efficiently utilized in the term structure model, the joint estimation shrinks the confidence interval to a much narrower range. As a result, the left panel shows clearly that the previously insignificant impacts for the very young before school years and old ages above 78 become significantly negative and positive, respectively; the uncertainty concerning the implied trend is also much reduced, especially between mid 1970s and mid 1980s around the turning point at the peak.

Figure 7 shows the resulting decomposition of yields into trends and the cyclical components. As can be seen, driven by the expected impact of current to future demographic age distribution, the trends in long-term yields also trace very well their low-frequency movements. The remaining cyclical components all pass the ADF stationarity test.

\subsection{Term structure decomposition}

The trends form a demography-driven term structure, as plotted in Figure 8. It is evident how the expected movement of demographic age distribution actually shapes the slow- 

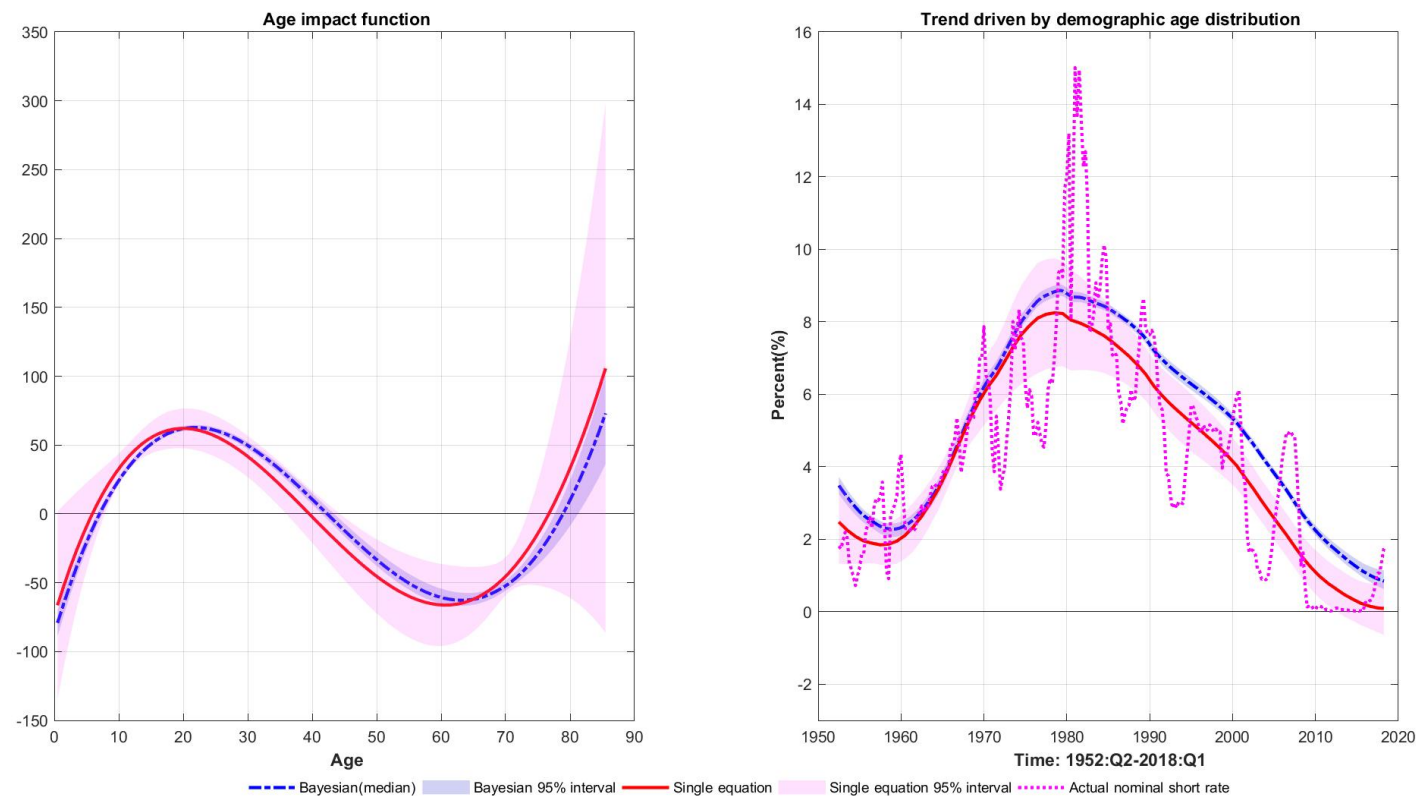

Figure 6. Age impact functions and trends from the joint model versus single equation regression
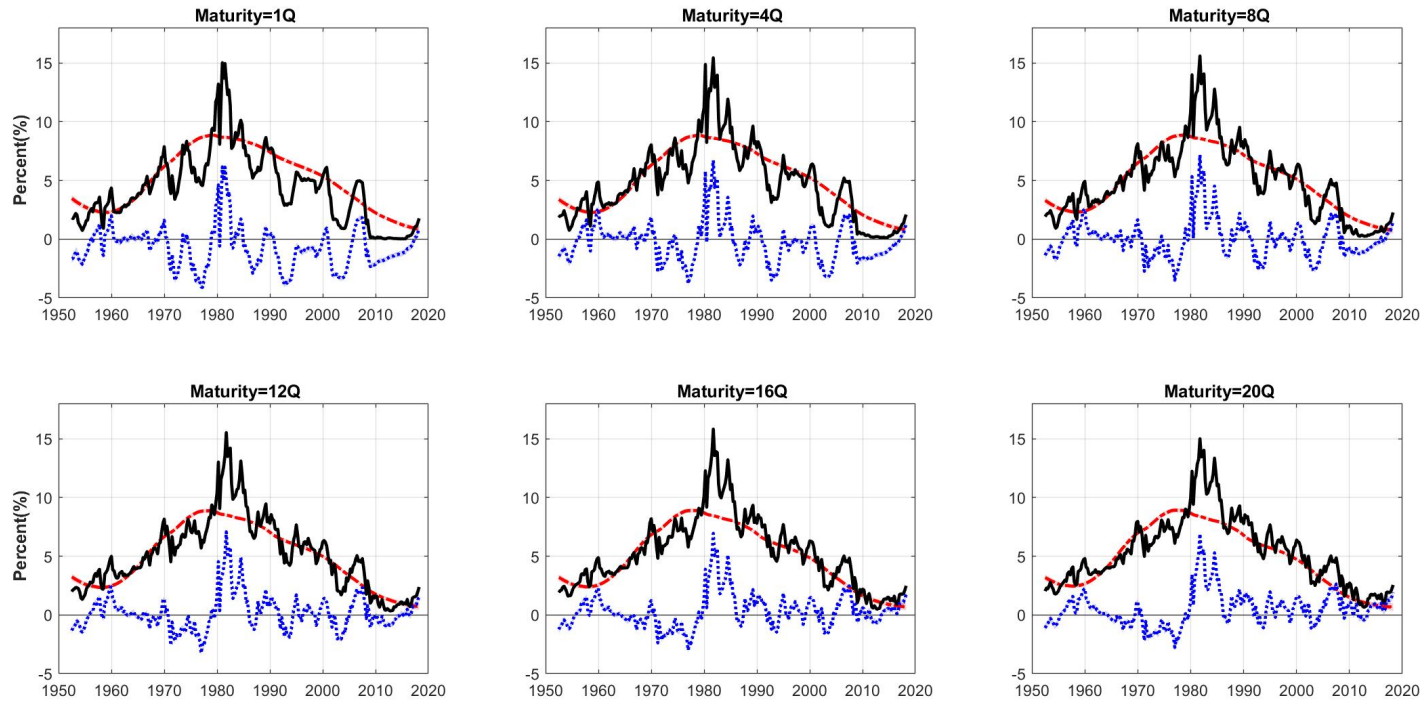

Figure 7. Yields decomposition in trends and cycles from the joint model

moving term structure in different stages, upward sloping during 1960s and up to mid 1970s due to high birth rate of the baby boomers and their growing impact on the interest rate when they are young, downward sloping from 1980s onwards for more than three decades as the baby boomers mature and age, and being flat during the transitional periods such as the latter half of 1970s and the recent few years. The term spread 
determined by the expected demography-driven trend can be as much as 70 bps on the upward trend with a positive slope and around 100 bps on the downward track with a negative slope.

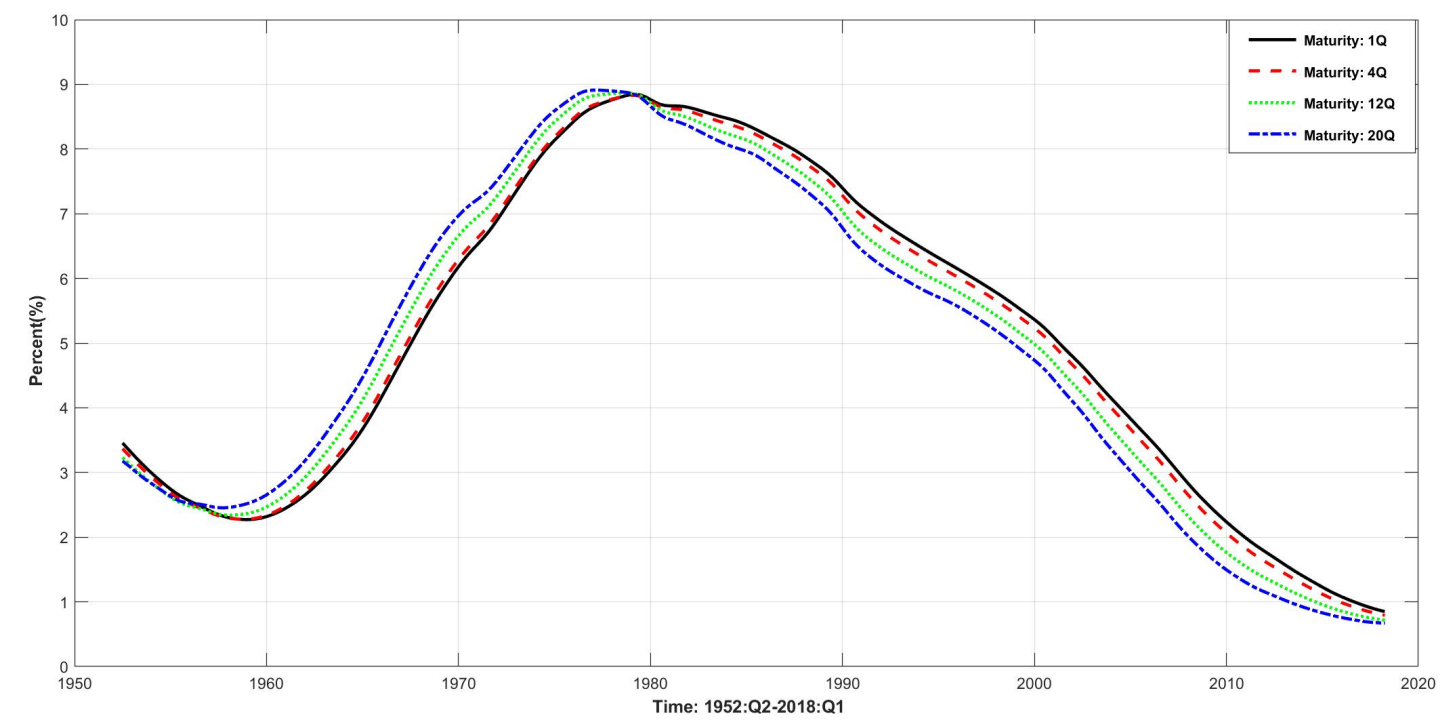

Figure 8. Demography-driven term structure of interest rates

Conditional on the slow-moving demography-driven term structure, there is a cyclical term structure remained in the yield curve, as plotted in Figure 9, which is stationary and less volatile. From the statistics in (Table 4), which compares the actual term structure and its two components, one can see that the average term spread of 5-year minus 3month yield in this cyclical term structure is $1.253 \%, 16.6$ bps higher than the whole yield difference, and the variance of the cyclical yields is about $50 \%$ less than that of the whole yields. The higher term spread in the cyclical components implies that the risk premia counts more in long-term yields during the sample, after controlling for the trend structure.

To see the model-implied dynamics of risk premia displayed in Figure 10, the 5-year yields are decomposed into the demography-driven expectation (trend), expectation of the cyclical component (expected average of cyclical short-term yield in the future horizons), and the risk premium. The first two terms can be combined as a total expectation term. It can be seen that now the expectation term dominates in the 5-year yield. The expected 


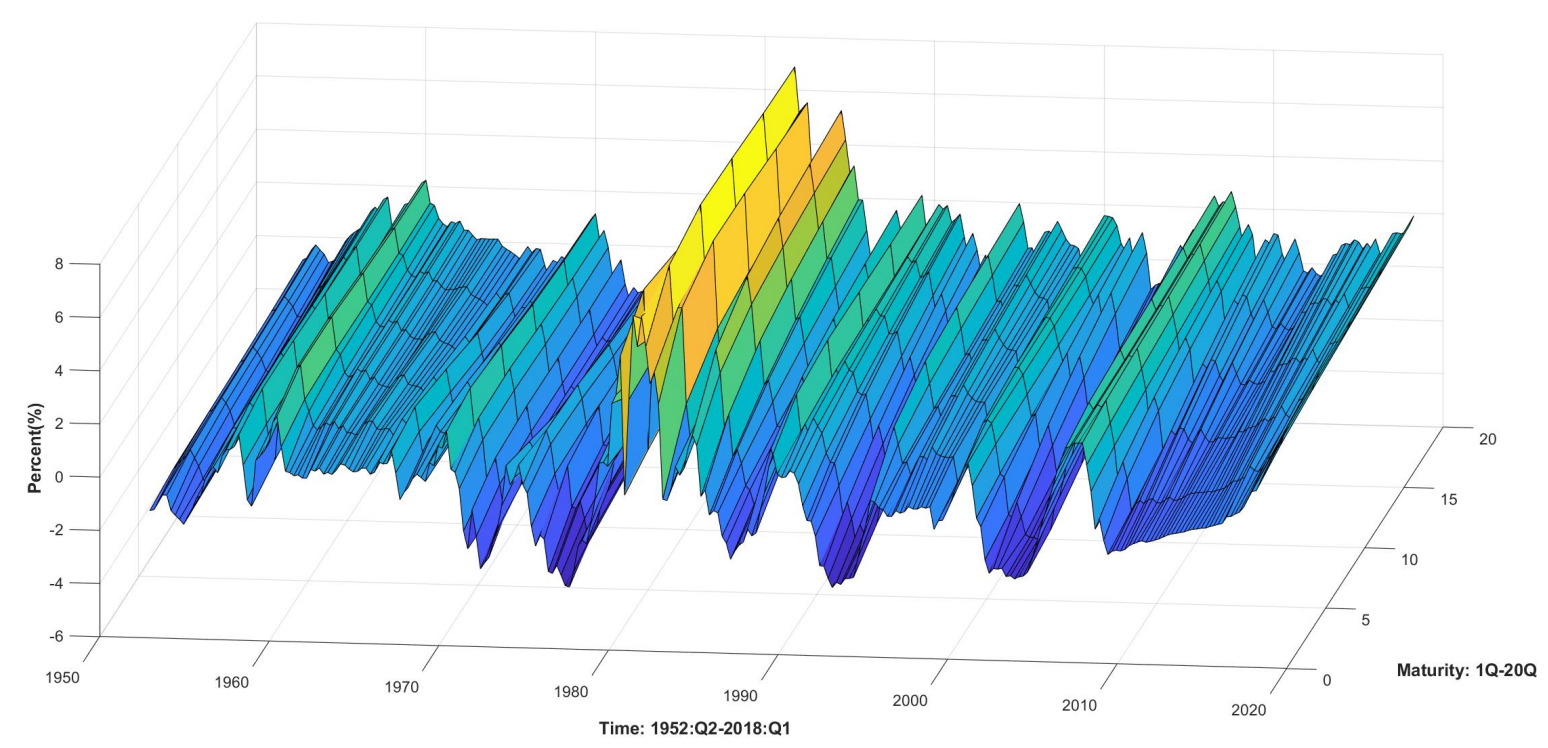

Figure 9. Cyclical term structure of interest rates

Table 4. Statistics of the decomposed term structure (\%)

\begin{tabular}{ccccccc}
\hline & \multicolumn{2}{c}{ Yields } & \multicolumn{2}{c}{ Trends } & \multicolumn{2}{c}{ Cycles } \\
\hline Maturity & Mean & Std & Mean & Std & Mean & Std \\
\hline 1Q & 4.317 & 3.090 & 5.064 & 2.530 & -0.741 & 1.719 \\
\hline 4Q & 4.757 & 3.201 & 5.031 & 5.011 & 2.547 & 1.682 \\
\hline $8 \mathrm{Q}$ & 4.957 & 3.166 & 4.982 & 2.570 & 0.006 & 1.569 \\
\hline $12 \mathrm{Q}$ & 5.140 & 3.088 & 4.955 & 2.589 & 0.180 & 1.488 \\
\hline $16 \mathrm{Q}$ & 5.294 & 3.030 & 4.929 & 2.606 & 0.342 & 1.455 \\
\hline $20 \mathrm{Q}$ & 5.404 & 2.959 & 4.904 & 2.619 & 0.513 & 1.451 \\
\hline $20 \mathrm{Q}-1 \mathrm{Q}$ & 1.087 & 1.000 & -0.161 & 0.557 & 1.253 & 1.239 \\
\hline
\end{tabular}

Note: This table shows the mean and standard deviation of actual yields, and median trends and cycles in yields.

yield almost always declines during a recession, and the risk premia fluctuates more than the cyclical expectation term. In comparison, if the 5-year risk premia is computed from an AFNS model without controlling the demographic trend, the risk premium contributes more in the upward trending period before end 1970s and counts less in the downward trending period, especially during the last ten years.

Taking the model-implied risk premia and computing their correlation with real activity indicators, such as IP growth and real consumption growth, Table 5 shows that the risk premia from our model negatively correlated with these macroeconomic variables with a counter-cyclical feature as predicted by theory. The premia from AFNS model 



Figure 10. Decomposition of 5-year yield

does not show such a feature.

Table 5. Correlation of risk premia and real activity

\begin{tabular}{|c|c|c|c|c|c|c|}
\hline Real activity index & Models & $4 \mathrm{Q}$ & $8 Q$ & $12 \mathrm{Q}$ & $16 \mathrm{Q}$ & $20 \mathrm{Q}$ \\
\hline \multirow{4}{*}{ IP growth } & \multirow{2}{*}{ SP-AFNS } & 0.009 & -0.057 & -0.106 & -0.136 & -0.152 \\
\hline & & $(-0.040,0.059)$ & $(-0.108,0.003)$ & $(-0.148,-0.049)$ & $(-0.170,-0.088)$ & $(-0.182,-0.113)$ \\
\hline & \multirow{2}{*}{ AFNS } & 0.051 & 0.012 & -0.017 & -0.034 & -0.042 \\
\hline & & $(0.011,0.093)$ & $(-0.034,0.060)$ & $(-0.063,0.033)$ & $(-0.079,0.014)$ & $(-0.088,0.001)$ \\
\hline \multirow{4}{*}{ Real consumption growth } & \multirow{2}{*}{ SP-AFNS } & 0.078 & -0.019 & -0.096 & -0.146 & -0.176 \\
\hline & & $(0.013,0.143)$ & $(-0.088,0.069)$ & $(-0.155,-0.006)$ & $(-0.192,-0.066)$ & $(-0.215,-0.109)$ \\
\hline & \multirow{2}{*}{ AFNS } & 0.159 & 0.124 & 0.093 & 0.072 & 0.059 \\
\hline & & $(0.124,0.194)$ & $(0.080,0.168)$ & $(0.047,0.143)$ & $(0.027,0.122)$ & $(0.015,0.106)$ \\
\hline \multirow{4}{*}{ Real GDP growth } & \multirow{2}{*}{ SP-AFNS } & 0.084 & 0.001 & -0.065 & -0.107 & -0.133 \\
\hline & & $(0.026,0.141)$ & $(-0.065,0.076)$ & $(-0.123,0.011)$ & $(-0.156,-0.041)$ & $(-0.175,-0.077)$ \\
\hline & \multirow{2}{*}{ AFNS } & 0.139 & 0.093 & 0.058 & 0.037 & 0.026 \\
\hline & & $(0.093,0.182)$ & $(0.033,0.149)$ & $(-0.010,0.119)$ & $(-0.034,0.097)$ & $(-0.049,0.083)$ \\
\hline
\end{tabular}

Note: This table shows the median of correlations between risk premia and real activity index, and 95\% confidence interval in the parenthesis consisting of $2.5 \%$ and $97.5 \%$ quantile of correlation.

\subsection{Comparison of Forecasting}

We compare the out-of-sample forecast of our model with a few alternatives, including a discrete-time AFNS as in Christensen et al. (2011) and Hong et al. (2019), dynamic Nelson-Siegel model (DNS) without no-arbitrage restrictions in Diebold and Li (2006), 
the single index demographic trend no-arbitrage model (MY-AFNS) in the spirit of Favero et al. (2016), and a random walk model without drift.

We run recursive window estimation, with the first window starting from 1952-Q2 to 1999-Q4 for 1-, 4-, 8-, 12-, 16-, and 20-quarter ahead forecast, and move one quarter a time with an expanding window for the next set of forecasts from 1- to 20-quarter ahead, until reaching the end of the sample. The results of root mean squared forecast errors (RMSFE) are reported in Figure 11, from which it can be seen that no alternative models beat our SP-AFNS model for the 4- to 20-quarter ahead forecast.
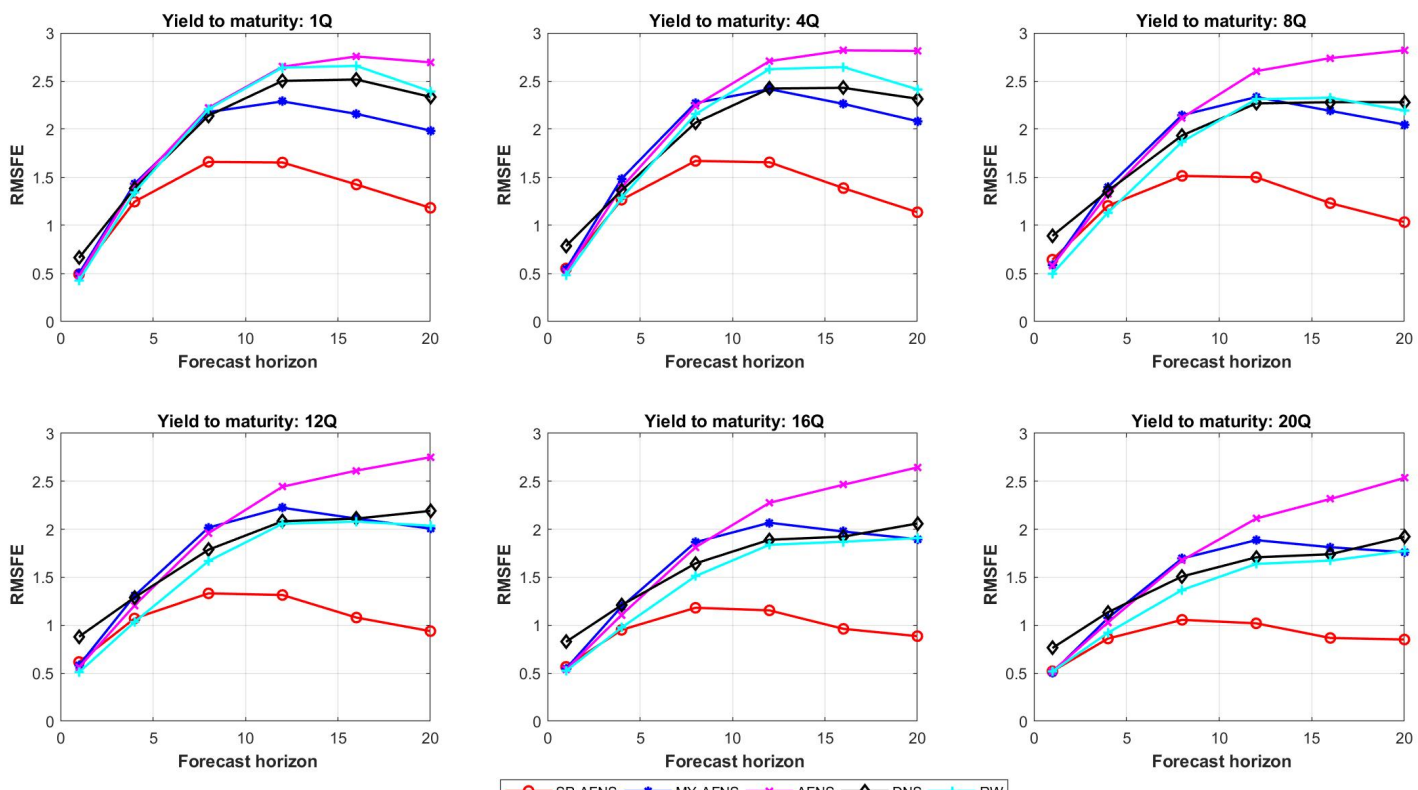

Figure 11. Forecast comparison based on RMSFE

Moreover, the error terms in alternative models increase with forecast horizons, but errors in the SP-AFNS model exhibit a slightly hump-shaped pattern, increase up to 12-quarter horizon and decrease afterwards, such that the errors in 20-quarter stabilized at around 150 bps across maturities. This non-diverging pattern of errors along forecast horizons is consistent with the unbiasedness of the trend prediction.

Finally, in Table 6, the advantage of the proposed model is also confirmed by a modified robust version of Diebold-Mariano test in Diebold and Mariano (1995) as in Harvey et al. (1997), where the negative terms indicate favorable results against the alternative models. 
Table 6. Predictability comparison based on Diebold-Mariano test

\begin{tabular}{|c|c|c|c|c|c|c|c|}
\hline \multicolumn{2}{|c|}{ Forecasting horizons } & $\mathrm{h}=1 \mathrm{Q}$ & $\mathrm{h}=4 \mathrm{Q}$ & $\mathrm{h}=8 \mathrm{Q}$ & $\mathrm{h}=12 \mathrm{Q}$ & $\mathrm{h}=16 \mathrm{Q}$ & $\mathrm{h}=20 \mathrm{Q}$ \\
\hline \multicolumn{2}{|c|}{ Competing models } & \multicolumn{6}{|c|}{ SP-AFNS vs. MY-AFNS } \\
\hline \multirow{6}{*}{ Maturities } & $1 \mathrm{Q}$ & -0.418 & -1.116 & -1.459 & -1.522 & $-1.824^{*}$ & -1.398 \\
\hline & $4 \mathrm{Q}$ & 0.345 & -1.590 & $-1.713^{*}$ & $-1.794^{*}$ & $-2.081^{* *}$ & -1.486 \\
\hline & $8 Q$ & $2.810^{* *}$ & $-1.697^{*}$ & $-1.908^{*}$ & $-2.004^{* *}$ & $-2.264^{* *}$ & -1.590 \\
\hline & $12 \mathrm{Q}$ & $2.151^{* *}$ & $-1.868^{*}$ & $-1.950^{*}$ & $-2.046^{* *}$ & $-2.286^{* *}$ & -1.620 \\
\hline & $16 \mathrm{Q}$ & 1.249 & $-1.768^{*}$ & $-1.891^{*}$ & $-2.001^{* *}$ & $-2.231^{* *}$ & -1.563 \\
\hline & $20 \mathrm{Q}$ & 0.844 & -1.519 & $-1.814^{*}$ & $-1.966^{*}$ & $-2.206^{* *}$ & -1.469 \\
\hline \multicolumn{2}{|c|}{ Competing models } & \multicolumn{6}{|c|}{ SP-AFNS vs. AFNS } \\
\hline \multirow{6}{*}{ Maturities } & $1 \mathrm{Q}$ & 1.133 & -0.967 & $-1.676^{*}$ & $-2.118^{* *}$ & $-3.104^{* * *}$ & $-2.507^{* *}$ \\
\hline & $4 \mathrm{Q}$ & $2.291^{* *}$ & -0.954 & $-1.839^{*}$ & $-2.229^{* *}$ & $-3.003^{* * *}$ & $-2.674^{* * *}$ \\
\hline & $8 \mathrm{Q}$ & $3.331^{* * *}$ & -0.884 & $-1.861^{*}$ & $-2.204^{* *}$ & $-2.721^{* * *}$ & $-2.739^{* * *}$ \\
\hline & $12 \mathrm{Q}$ & $2.754^{* * *}$ & -0.908 & $-1.833^{*}$ & $-2.164^{* *}$ & $-2.603^{* *}$ & $-2.827^{* * *}$ \\
\hline & $16 \mathrm{Q}$ & 1.355 & -0.987 & $-1.771^{*}$ & $-2.121^{* *}$ & $-2.544^{* *}$ & $-2.933^{* * *}$ \\
\hline & $20 \mathrm{Q}$ & 0.033 & -1.014 & -1.714 & $-2.131^{* *}$ & $-2.623^{* *}$ & $-3.329^{* * *}$ \\
\hline \multicolumn{2}{|c|}{ Competing models } & \multicolumn{6}{|c|}{ SP-AFNS vs. DNS } \\
\hline \multirow{6}{*}{ Maturities } & $1 \mathrm{Q}$ & $-5.365^{* * *}$ & -0.839 & -1.374 & -1.613 & $-1.767^{*}$ & $-2.107^{*}$ \\
\hline & $4 \mathrm{Q}$ & $-5.800^{* * *}$ & -0.993 & -1.462 & $-1.710^{*}$ & $-1.804^{*}$ & $-2.107^{* *}$ \\
\hline & $8 \mathrm{Q}$ & $-6.014^{* * *}$ & $-1.753^{*}$ & -1.637 & $-1.772^{*}$ & $-1.800^{*}$ & $-2.089^{* *}$ \\
\hline & $12 \mathrm{Q}$ & $-6.144^{* * *}$ & $-2.230^{* *}$ & $-1.759^{*}$ & $-1.798^{*}$ & $-1.765^{*}$ & $-2.053^{* *}$ \\
\hline & $16 \mathrm{Q}$ & $-5.566^{* * *}$ & $-2.311^{* *}$ & $-1.774^{*}$ & $-1.766^{*}$ & $-1.708^{*}$ & $-2.001^{*}$ \\
\hline & $20 \mathrm{Q}$ & $-4.850^{* * *}$ & $-2.282^{* *}$ & $-1.760^{*}$ & $-1.734^{*}$ & $-1.681^{*}$ & $-1.999^{*}$ \\
\hline \multicolumn{2}{|c|}{ Competing models } & \multicolumn{6}{|c|}{ SP-AFNS vs. Random walk } \\
\hline \multirow{6}{*}{ Maturities } & $1 \mathrm{Q}$ & $2.799^{* *}$ & -0.573 & $-1.706^{*}$ & $-1.807^{*}$ & $-1.813^{*}$ & $-2.791^{* * *}$ \\
\hline & $4 \mathrm{Q}$ & $1.828^{*}$ & -0.127 & $-1.937^{*}$ & $-2.000^{* *}$ & $-1.916^{*}$ & $-3.324^{* * *}$ \\
\hline & $8 \mathrm{Q}$ & $3.482^{* * *}$ & 0.586 & $-1.862^{*}$ & $-2.027^{* *}$ & $-1.982^{*}$ & $-4.125^{* * *}$ \\
\hline & $12 \mathrm{Q}$ & $2.671^{* * *}$ & 0.384 & $-1.879^{*}$ & $-2.049^{* *}$ & $-2.049^{* *}$ & $-4.101^{* * *}$ \\
\hline & $16 \mathrm{Q}$ & 1.310 & -0.280 & $-1.886^{*}$ & $-2.031^{* *}$ & $-2.024^{* *}$ & $-3.661^{* * *}$ \\
\hline & $20 \mathrm{Q}$ & 0.069 & -0.829 & $-1.782^{*}$ & $-1.935^{*}$ & $-1.971^{*}$ & $-3.593^{* * *}$ \\
\hline
\end{tabular}

\section{Conclusion}

We propose a unified semi-parametric affine term structure modeling framework, termed as SP-ATSM models, to incorporate a long-run trend driven by the demographic age distribution through a life-cycle impact function. On one hand, the information in the term structure is efficiently utilized to infer the age impact function with tightness on the parameters, and the resulting age impact function shows a life-cycle pattern consistent with the impact through real rate and inflation trends in a Fisherian equation framework. On the other hand, the strong identification on a slow-moving trend as the integrated effect of the evolving age distributions helps to decompose the interest rate term structure into 
a demography-driven structure and a cyclical one.

Applying the proposed model to study the U.S. data from 1950 to present, we find remarkable evidence on the consistency of our model with respect to the yield data. The cyclical term structure is stationary and the implied risk premia is counter-cyclical, as predicted by theory. Out-of-sample prediction from the model beats alternatives for 4- to 20-quarter ahead forecast for yields of all maturities.

Finally, some extensions of the proposed model into a joint framework with inflation protected bond yields may help to better pin down the life-cycle age impact functions of inflation and real rate and they are warranted as a future research.

\section{References}

Abel, A. B. (2003). The effects of a baby boom on stock prices and capital accumulation in the presence of social security. Econometrica 71 (2), 551-578.

Aksoy, Y., H. S. Basso, R. P. Smith, and T. Grasl (2019). Demographic structure and macroeconomic trends. American Economic Journal: Macroeconomics 11(1), 193-222.

Andrews, D. W. (1991). Asymptotic normality of series estimators for nonparametric and semiparametric regression models. Econometrica 59(1), 307-345.

Ang, A. and G. Bekaert (2002). Regime switches in interest rates. Journal of Business $\mathscr{E}$ Economic Statistics 20(2), 163-182.

Ang, A., J. Boivin, S. Dong, and R. Loo-Kung (2011). Monetary policy shifts and the term structure. Review of Economic Studies 78(2), 429-457.

Ang, A., S. Dong, and M. Piazzesi (2007). No-arbitrage Taylor rules. Technical report, National Bureau of Economic Research.

Ang, A. and A. Maddaloni (2005). Do demographic changes affect risk premiums? evidence from international data. Journal of Business 78(1), 341-380. 
Ang, A. and M. Piazzesi (2003). A no-arbitrage vector autoregression of term structure dynamics with macroeconomic and latent variables. Journal of Monetary economics $50(4)$, $745-787$.

Bakshi, G. S. and Z. Chen (1994). Baby boom, population aging, and capital markets. Journal of Business 67(1), 165-202.

Bauer, M. D. and G. D. Rudebusch (2020). Interest rates under falling stars. American Economic Review 110(5), 1316-54.

Bloom, D. E., D. Canning, and B. Graham (2003). Longevity and life-cycle savings. Scandinavian Journal of Economics 105(3), 319-338.

Bowsher, C. G. and R. Meeks (2008). The dynamics of economic functions: modeling and forecasting the yield curve. Journal of the American Statistical Association 103(484), $1419-1437$.

Browning, M. and M. Ejrnæs (2009). Consumption and children. Review of Economics and Statistics 91(1), 93-111.

Bullard, J., C. Garriga, C. J. Waller, et al. (2012). Demographics, redistribution, and optimal inflation. Federal Reserve Bank of St. Louis Review 94(6), 419-39.

Burman, P., E. Chow, and D. Nolan (1994). A cross-validatory method for dependent data. Biometrika $81(2), 351-358$.

Carter, C. K. and R. Kohn (1994). On gibbs sampling for state space models. Biometrika 81(3), 541-553.

Chen, Y. and L. Niu (2014). Adaptive dynamic Nelson-Siegel term structure model with applications. Journal of Econometrics 180(1), 98-115.

Chib, S. and B. Ergashev (2009). Analysis of multifactor affine yield curve models. Journal of the American Statistical Association 104(488), 1324-1337. 
Christensen, J. H., F. X. Diebold, and G. D. Rudebusch (2011). The affine arbitrage-free class of nelson-siegel term structure models. Journal of Econometrics 164(1), 4-20.

Cieslak, A. and P. Povala (2015). Expected returns in treasury bonds. Review of Financial Studies 28(10), 2859-2901.

Cocco, J. F. and F. J. Gomes (2012). Longevity risk, retirement savings, and financial innovation. Journal of Financial Economics 103(3), 507-529.

Cox, J. C., J. E. Ingersoll, and S. A. Ross (1985). A theory of the term structure of interest rates. Econometrica 53(2), 385-407.

Del Negro, M., D. Giannone, M. P. Giannoni, and A. Tambalotti (2019). Global trends in interest rates. Journal of International Economics 118(1), 248-262.

DellaVigna, S. and J. M. Pollet (2007). Demographics and industry returns. American Economic Review 97(5), 1667-1702.

Diebold, F. X. and C. Li (2006). Forecasting the term structure of government bond yields. Journal of Econometrics 130(2), 337-364.

Diebold, F. X. and R. S. Mariano (1995). Comparing predictive accuracy. Journal of Business \& Economic Statistics $13(3)$.

Eggertsson, G. B., N. R. Mehrotra, and J. A. Robbins (2019). A model of secular stagnation: Theory and quantitative evaluation. American Economic Journal: Macroeconomics $11(1), 1-48$.

Fair, R. C. and K. M. Dominguez (1991). Effects of the changing us age distribution on macroeconomic equations. American Economic Review 81(5), 1276-1294.

Fama, E. F. (2006). The behavior of interest rates. Review of Financial Studies 19(2), $359-379$.

Favero, C. A., A. E. Gozluklu, and A. Tamoni (2011). Demographic trends, the dividendprice ratio, and the predictability of long-run stock market returns. Journal of Financial and Quantitative Analysis 46(5), 1493-1520. 
Favero, C. A., A. E. Gozluklu, and H. Yang (2016). Demographics and the behavior of interest rates. IMF Economic Review 64(4), 732-776.

Geanakoplos, J., M. Magill, and M. Quinzii (2004). Demography and the long-run predictability of the stock market. Brookings Papers on Economic Activity 2004(1), 241325.

Ghysels, E. and S. Ng (1998). A semiparametric factor model of interest rates and tests of the affine term structure. Review of Economics and Statistics 80(4), 535-548.

Goliński, A. and P. Zaffaroni (2016). Long memory affine term structure models. Journal of Econometrics $191(1), 33-56$.

Goyal, A. (2004). Demographics, stock market flows, and stock returns. Journal of Financial and Quantitative Analysis 39(1), 115-142.

Härdle, W. K. and P. Majer (2016). Yield curve modeling and forecasting using semiparametric factor dynamics. European Journal of Finance 22(12), 1109-1129.

Harvey, D., S. Leybourne, and P. Newbold (1997). Testing the equality of prediction mean squared errors. International Journal of forecasting 13(2), 281-291.

Higgins, M. (1998). Demography, national savings, and international capital flows. International Economic Review 39(2), 343-369.

Hong, Z., L. Niu, and G. Zeng (2019). US and Chinese yield curve responses to rmb exchange rate policy shocks. China Finance Review International 9(3), 360-385.

Juselius, M. and E. Takáts (2015). Can demography affect inflation and monetary policy? BIS Working Papers Series No 485, 1-44.

Katagiri, M., H. Konishi, and K. Ueda (2020). Aging and deflation from a fiscal perspective. Journal of Monetary Economics 111(1), 1-15.

Koop, G. (2003). Bayesian Econometrics. John Wiley \& Sons, New York. 
Kozicki, S. and P. A. Tinsley (2001). Shifting endpoints in the term structure of interest rates. Journal of Monetary Economics 47(3), 613-652.

Krueger, D. and A. Ludwig (2007). On the consequences of demographic change for rates of returns to capital, and the distribution of wealth and welfare. Journal of Monetary Economics 54(1), 49-87.

Lindh, T. and B. Malmberg (2000). Can age structure forecast inflation trends? Journal of Economics and Business 52(1-2), 31-49.

Love, D. A. (2010). The effects of marital status and children on savings and portfolio choice. Review of Financial Studies 23(1), 385-432.

McMillan, H. M. and J. B. Baesel (1990). The macroeconomic impact of the baby boom generation. Journal of Macroeconomics 12(2), 167-195.

Modigliani, F. (1966). The life cycle hypothesis of saving, the demand for wealth and the supply of capital. Social Research, 160-217.

Newey, W. K. and K. D. West (1987). A simple, positive semi-definite, heteroskedasticity and autocorrelation. Econometrica 55(3), 703-708.

Park, C. (2010). How does changing age distribution impact stock prices? a nonparametric approach. Journal of Applied Econometrics 25(7), 1155-1178.

Park, J. Y., K. Shin, and Y.-J. Whang (2010). A semiparametric cointegrating regression: Investigating the effects of age distributions on consumption and saving. Journal of Econometrics 157(1), 165-178.

Poterba, J. M. (2001). Demographic structure and asset returns. Review of Economics and Statistics 83(4), 565-584.

Racine, J. (1997). Feasible cross-validatory model selection for general stationary processes. Journal of Applied Econometrics 12(2), 169-179.

Rasay, J. O. and B. W. Silverman (1997). Functional Data Analysis. Springer, New York. 
Vasicek, O. (1977). An equilibrium characterization of the term structure. Journal of Financial Economics 5(2), 177-188.

Zhang, J., J. Zhang, and R. Lee (2003). Rising longevity, education, savings, and growth. Journal of Development Economics 70(1), 83-101. 